\title{
Magellanic Clouds stellar clusters. II. New $B, V$ CM diagrams for 6 LMC and 10 SMC clusters ${ }^{\star}$
}

\author{
A. Matteucci ${ }^{1}$, V. Ripepi ${ }^{2}$, E. Brocato ${ }^{3}$, and V. Castellani ${ }^{1}$ \\ 1 Dipartimento di Fisica, Università di Pisa, Piazza Torricelli 2, 56100 Pisa, Italy \\ 2 Osservatorio Astronomico di Capodimonte, Via Moiariello 16, 80131 Napoli, Italy \\ 3 Osservatorio Astronomico di Collurania, Via M. Maggini, 64100 Teramo, Italy
}

Received 10 August 2001 / Accepted 29 January 2002

\begin{abstract}
We present new CCD photometry for 6 LMC and 10 SMC stellar clusters taken at the ESO 1.54-m Danish Telescope in La Silla, to extend a previous investigation on Magellanic Clouds clusters based on HST snapshots. Thanks to the much larger area covered by the Danish detector, we investigate the spatial distribution of cluster stars, giving $V,(B-V) \mathrm{CM}$ diagrams for both clusters and surrounding fields. Evidence of a complex history of star formation in the Clouds is outlined, showing that old field populations in both Clouds have metallicities much lower than normally adopted for them $(Z=0.008$ and $Z=0.004$ for LMC and SMC respectively), with SMC field stars more metal poor than in the LMC. Observational data concerning the red clump of field stars in both Clouds are briefly discussed.
\end{abstract}

Key words. galaxies: clusters: general - galaxies: stellar content - galaxies: Magellanic Clouds

\section{Introduction}

The Magellanic Clouds (MC) are well-known targets for the investigation of simple stellar populations, since they present a great number of populous young and intermediate age clusters, which allow to test evolutionary theories for a combination of masses and metallicities not present in our Galaxy. In a previous paper (Brocato et al. 2001) we have recently presented $B, V$ CMDs for 21 globular clusters in the LMC and 1 in the SMC, as obtained on the basis of HST snapshots. In this paper we extend this investigation by using observational data from the ESO 1.54-m Danish Telescope.

With respect to the previous survey, the present study obviously relies on a worse seeing, but presents also the relevant advantage of providing data in a larger field of view $\left(\sim 12 ! 5 \times 12^{\prime}\right)$, covering the whole cluster and a large portion of the surrounding stellar field. As we shall discuss later on, the quality of the CM diagrams presented in this paper appears largely comparable with the ones in the previous investigation. In this way we shall add 4 clusters to the previous sample of the LMC clusters (2 of our 6 LMC clusters were already investigated in the previous paper), extending the investigation to 10 clusters in the SMC in

Send offprint requests to: E. Brocato,

e-mail: brocato@te.astro.it

* Based on observations carried out at the European Southern Observatory, La Silla, Chile. order to allow the investigation of stellar populations in both Clouds.

Section 2 gives information on the observations and on the adopted data reduction procedures. The CM diagrams of clusters and surrounding fields are presented in Sect. 3 where the major features of the diagrams are briefly discussed. In Sect. 4 we discuss the stellar populations of the fields, whereas a study of the red clumps in both LMC and SMC fields is presented in Sect. 5. In Sect. 6 we finally present some brief concluding remarks.

\section{Observations, data reduction and calibration}

$B$ (ESO \# 450 filter) and $V$ (ESO \# 451 filter) images were collected by one of us (E. B.) during five nights, from Nov. 28 to Dec. 21997 (we shall refer to the nights as $\mathrm{N} 1$ to N5, where N1 = Nov. 28 night), with the ESO 1.54-m Danish Telescope (La Silla, Chile). The instrument installed at the telescope was the DFOSC, equipped with a LORAL/LESSER C1W7 CCD (2052 × 2052 pixels). The scale was $0.39 \mathrm{arcsec} /$ pixel. The log of observations is reported in Table 1.

The original images were pre-reduced following the usual procedures, i.e. bias subtraction and flat-fielding. However, the edges of the frames have been trimmed to remove the overscan columns and the regions of the CCD where flat-fielding showed dramatic differences with respect to the central part of the CCD image. The resulting 
Table 1. Log of the observations.

\begin{tabular}{|c|c|c|c|c|c|}
\hline $\begin{array}{l}\text { Cluster } \\
\text { (Galaxy) }\end{array}$ & Filter & $\begin{array}{c}\text { Observation } \\
\text { night }\end{array}$ & $\begin{array}{c}\text { Number of } \\
\text { observations }\end{array}$ & $\begin{array}{c}\text { Min, max exposure } \\
\text { time }(\mathrm{s})\end{array}$ & $\begin{array}{l}\text { Seeing } \\
(\operatorname{arcsec})\end{array}$ \\
\hline KMHK $264^{\mathrm{a}}$ & $B$ & $\mathrm{~N} 4$ & 2 & 60,300 & $1.3-1.4$ \\
\hline (LMC) & $V$ & $\mathrm{~N} 4$ & 2 & 30,200 & $1.2-1.3$ \\
\hline NGC 1777 & $B$ & N1 & 5 & 120,300 & $1.6-1.8$ \\
\hline (LMC) & $V$ & N1 & 5 & 60,200 & $1.6-2.0$ \\
\hline NGC 1902 & $B$ & $\mathrm{~N} 1, \mathrm{~N} 4, \mathrm{~N} 5$ & 4 & 40,300 & $1.2-1.8$ \\
\hline (LMC) & $V$ & $\mathrm{~N} 1, \mathrm{~N} 4, \mathrm{~N} 5$ & 4 & 20,200 & $1.1-1.6$ \\
\hline NGC 2155 & $B$ & N3 & 3 & 60,600 & 1.2 \\
\hline (LMC) & $V$ & N3 & 4 & 30,300 & $1.1-1.2$ \\
\hline IC 2146 & $B$ & $\mathrm{~N} 4$ & 3 & 60,1200 & $1.2-1.3$ \\
\hline (LMC) & $V$ & N4 & 4 & 30,600 & 1.2 \\
\hline L9 & $B$ & N5 & 3 & 60,600 & $1.3-1.4$ \\
\hline$(\mathrm{SMC})$ & $V$ & N5 & 4 & 30,300 & $1.1-1.4$ \\
\hline L13 & $B$ & N5 & 2 & 120,600 & $1.3-1.4$ \\
\hline (SMC) & $V$ & N5 & 3 & 60,300 & $1.2-1.4$ \\
\hline $\mathrm{L} 45^{\mathrm{b}}$ & $B$ & N5 & 3 & 60,600 & $1.3-1.4$ \\
\hline (SMC) & $V$ & N5 & 3 & 30,300 & $1.3-1.4$ \\
\hline NGC 152 & $B$ & $\mathrm{~N} 4$ & 4 & 60,1200 & $1.2-1.4$ \\
\hline (SMC) & $V$ & $\mathrm{~N} 4$ & 5 & 30,600 & $1.1-1.2$ \\
\hline NGC $220^{\mathrm{c}}$ & $B$ & $\mathrm{~N} 1, \mathrm{~N} 2, \mathrm{~N} 4, \mathrm{~N} 5$ & 8 & 40,300 & $1.3-1.9$ \\
\hline (SMC) & $V$ & $\mathrm{~N} 1, \mathrm{~N} 2, \mathrm{~N} 4, \mathrm{~N} 5$ & 10 & 20,200 & $1.3-1.8$ \\
\hline NGC 458 & $B$ & $\mathrm{~N} 1, \mathrm{~N} 3, \mathrm{~N} 4, \mathrm{~N} 5$ & 5 & 60,300 & $1.2-1.9$ \\
\hline (SMC) & $V$ & N1, N3, N4, N5 & 5 & 30,300 & $1.1-1.8$ \\
\hline NGC 643 & $B$ & $\mathrm{~N} 2, \mathrm{~N} 3$ & 5 & 120,1200 & $1.2-2.2$ \\
\hline (SMC) & $V$ & $\mathrm{~N} 2, \mathrm{~N} 3$ & 12 & 30,600 & $1.1-2.3$ \\
\hline
\end{tabular}

a The same field of KMHK264 includes also the KMHK265 cluster.

b The same field of L45 includes also the NGC 299 cluster.

c The same field of NGC 220 includes also the NGC 222 and NGC 231 clusters.

frame dimensions were $1911 \times 1811$ pixels, for a total field of view of $\sim 12 ! 4 \times 11 ! 8$.

Photometry was performed using the point-spread function (PSF) technique with the DAOPHOT II package (Stetson 1994). Raw instrumental magnitudes and errors were obtained by fitting the PSF to all identified objects using the routine ALLSTAR (Stetson 1994). A detailed test of the resulting photometry revealed a region of the frames where stellar images appeared elongated because of optical aberrations. ALLSTAR deconvolved these objects into more stars than really present, providing a bad fit with high residuals. We eliminated from our star lists all the objects identified in this region, named as "Region 2" in Fig. 1. The field of view of the resulting "good" field (Region 1) is $\sim 113.4 \operatorname{arcmin}^{2}$.

For a given stellar field, each frame was reduced independently; then the frame with the best seeing was chosen in each filter as a reference for coordinate and magnitude transformations. Subsequently, all the frames where

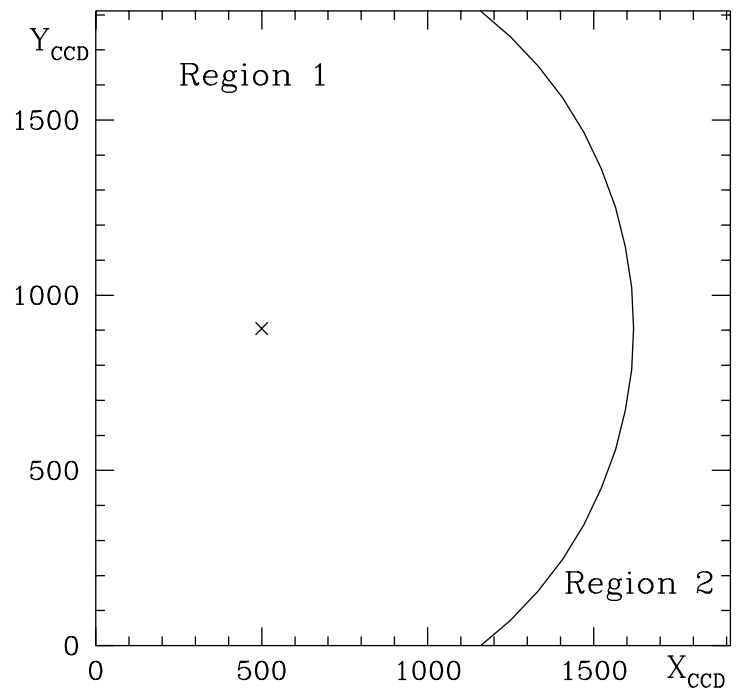

Fig. 1. CCD frame region (Region 2) affected by optical aberrations. The center of the dividing circumference is $(500,905)$ and its radius is 1120 pixels. 

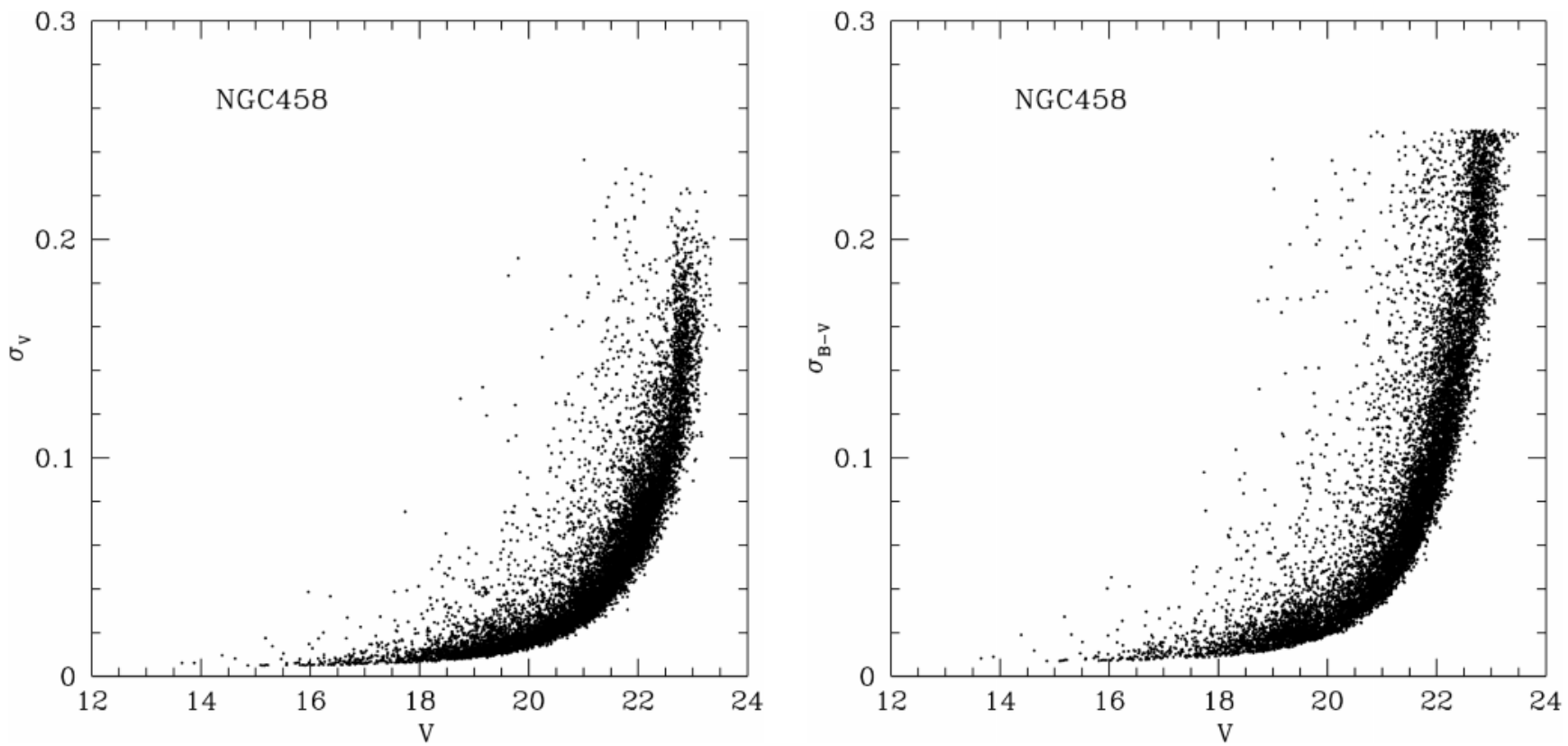

Fig. 2. Instrumental errors in magnitude $\left(\sigma_{V}\right)$ and colour $\left(\sigma_{B-V}\right)$ as a function of the calibrated magnitudes $(V)$ for the stars in the frames of NGC 458.

Table 2. Standard stars used for calibration.

\begin{tabular}{cccc}
\hline \hline Night & Reference field & Standard stars & $\begin{array}{c}\text { No. of } B, V \text { observations } \\
\text { per field }\end{array}$ \\
\hline \multirow{2}{*}{ N1 } & Selected Area 95 (Landolt 1992) & 42, 101, 105, 107 & 1,2 \\
& E3 Region (Graham 1982) & R & 1,1 \\
N3 & Selected Area 95 (Landolt 1992) & 42, 101, 105 & 1,1 \\
& E2 Region (Graham 1982) & I, m, o, s, t & 3,3 \\
& E3 Region (Graham 1982) & T, k, v & 1,1 \\
N4 & T Phe (Landolt 1992) & A, C, D, E, F & 6,6 \\
& E2 Region (Graham 1982) & I, m, o, s, t & 3,3 \\
N55 & T Phe (Landolt 1992) & A, C, D, E, F & 4,4 \\
& E2 Region (Graham 1982) & I, m, o, s, t & 2,2 \\
\hline
\end{tabular}

a Photometric night.

transformed into the reference system to perform suitable average operations in order to obtain a set of mean instrumental magnitudes (weighted according to the photometric quality of the frames). Finally, $B$ and $V$ photometry for each field were matched in order to obtain the instrumental magnitudes $(v)$, colours $(b-v)$ and errors (see Fig. 2).

To transform instrumental magnitude into the Johnson system, several standard stars from the E-regions (Graham 1982) and from equatorial fields (Landolt 1992) were observed every night, except N2 (see Table 2 for a detailed list of standards used); however, the best photometric night turned out to be N4.

We used calibrating relations of the form:

$$
V-v_{\mathrm{a}}=\alpha(b-v)_{\mathrm{a}}+\beta
$$$$
(B-V)=\gamma(b-v)_{\mathrm{a}}+\delta
$$

where $V,(B-V)$ indicate Johnson magnitude and colour, respectively, whereas $v_{\mathrm{a}}$ and $(b-v)_{\text {a }}$ are instrumental results corrected for atmospheric extinction and normalised to an exposure time of 1 second, as given by the following relations:

$$
\begin{aligned}
& b_{\mathrm{a}}=b+2.5 \log t-\epsilon_{B} X \\
& v_{\mathrm{a}}=v+2.5 \log t-\epsilon_{V} X
\end{aligned}
$$

where $\epsilon_{B}$ and $\epsilon_{V}$ are the adopted extinction coefficients and $X$ the airmass. Coefficients $\alpha, \beta, \gamma, \delta$ were found by a weighted least squares fit through the data on the N4 night, where the weights take into account both the errors on Landolt's and Graham's magnitudes and the errors in the aperture photometry. Figure 3 reports the calibration for the N4 night. 

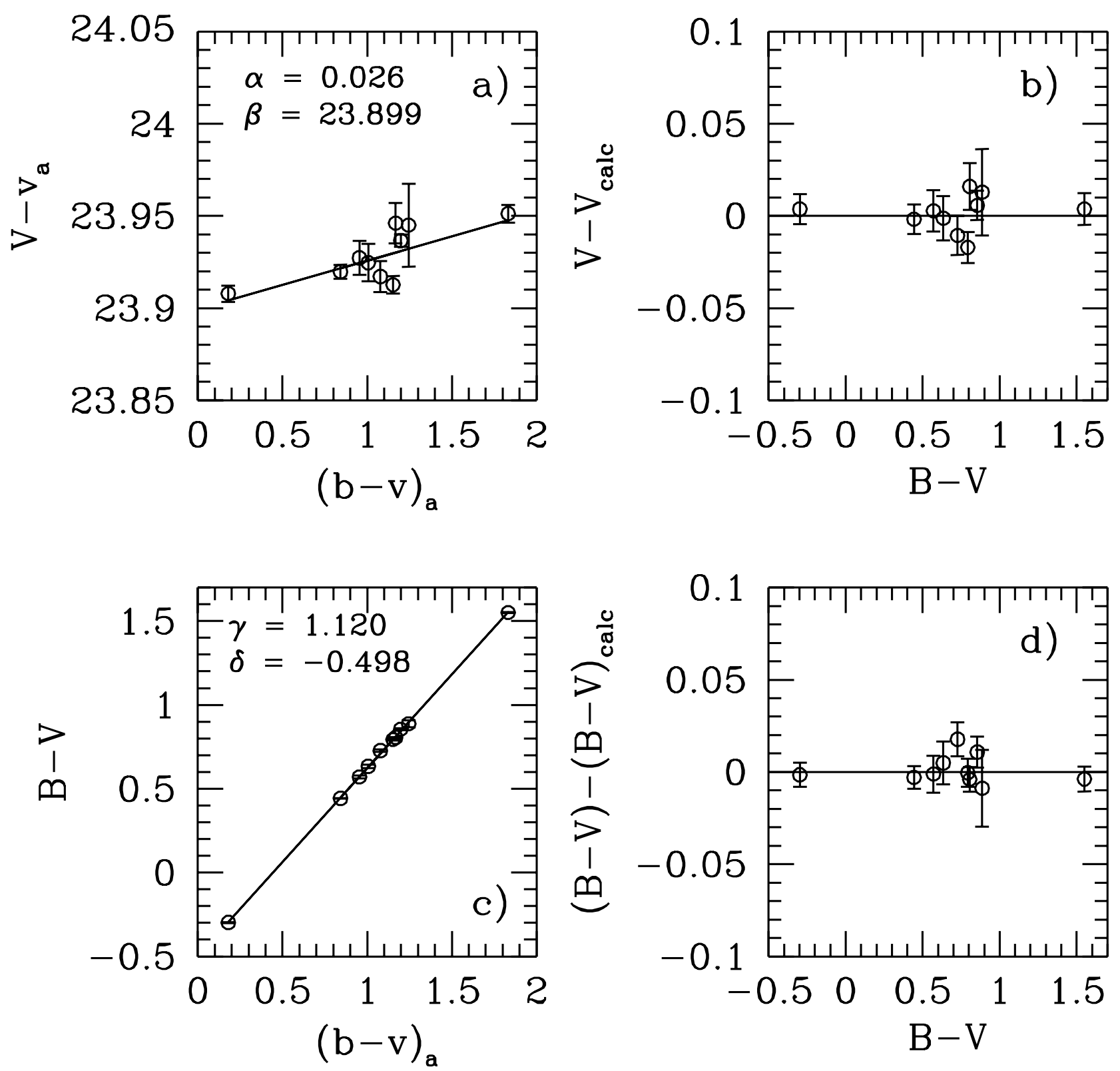

Fig. 3. Panels a), c): calibrating relations for N4 night, found by a weighted least squares fit. Panels b), d): difference between standard and calculated magnitudes and colours for the calibration stars; the result appears satisfactory.

Table 3. Colour and extinction coefficients and zero points (see Fig. 3) adopted to calibrate instrumental results; rms $V$, rms ${ }_{B-V}$ are the root mean squares from the fitting procedures; $\epsilon_{B}$ and $\epsilon_{V}$ are the adopted extinction coefficients in $B$ and $V$ bands, respectively.

\begin{tabular}{ccccccccc}
\hline \hline Night & $\alpha$ & $\beta$ & $\mathrm{rms}_{V}$ & $\gamma$ & $\delta$ & $\mathrm{rms}_{B-V}$ & $\epsilon_{B}$ & $\epsilon_{V}$ \\
\hline N1 & 0.026 & 23.912 & 0.014 & 1.120 & -0.485 & 0.021 & $0.20 \pm 0.01$ & $0.10 \pm 0.01$ \\
N3 & 0.026 & 23.913 & 0.013 & 1.120 & -0.487 & 0.010 & $0.20 \pm 0.01$ & $0.10 \pm 0.01$ \\
N4 & 0.026 & 23.899 & 0.008 & 1.120 & -0.498 & 0.006 & $0.198 \pm 0.007$ & $0.095 \pm 0.006$ \\
N5 & 0.026 & 23.902 & 0.006 & 1.120 & -0.486 & 0.009 & $0.20 \pm 0.01$ & $0.095 \pm 0.006$ \\
\hline
\end{tabular}

To obtain internal consistence among our data from different nights, we used the SMC cluster NGC458, which was observed during each night. We adopted the $\alpha$ and $\gamma$ colour coefficients of N4 also for N1, N3, and N5 and determined new zero points, reaching a good level of consistence (residual discrepancies from one night to the other 

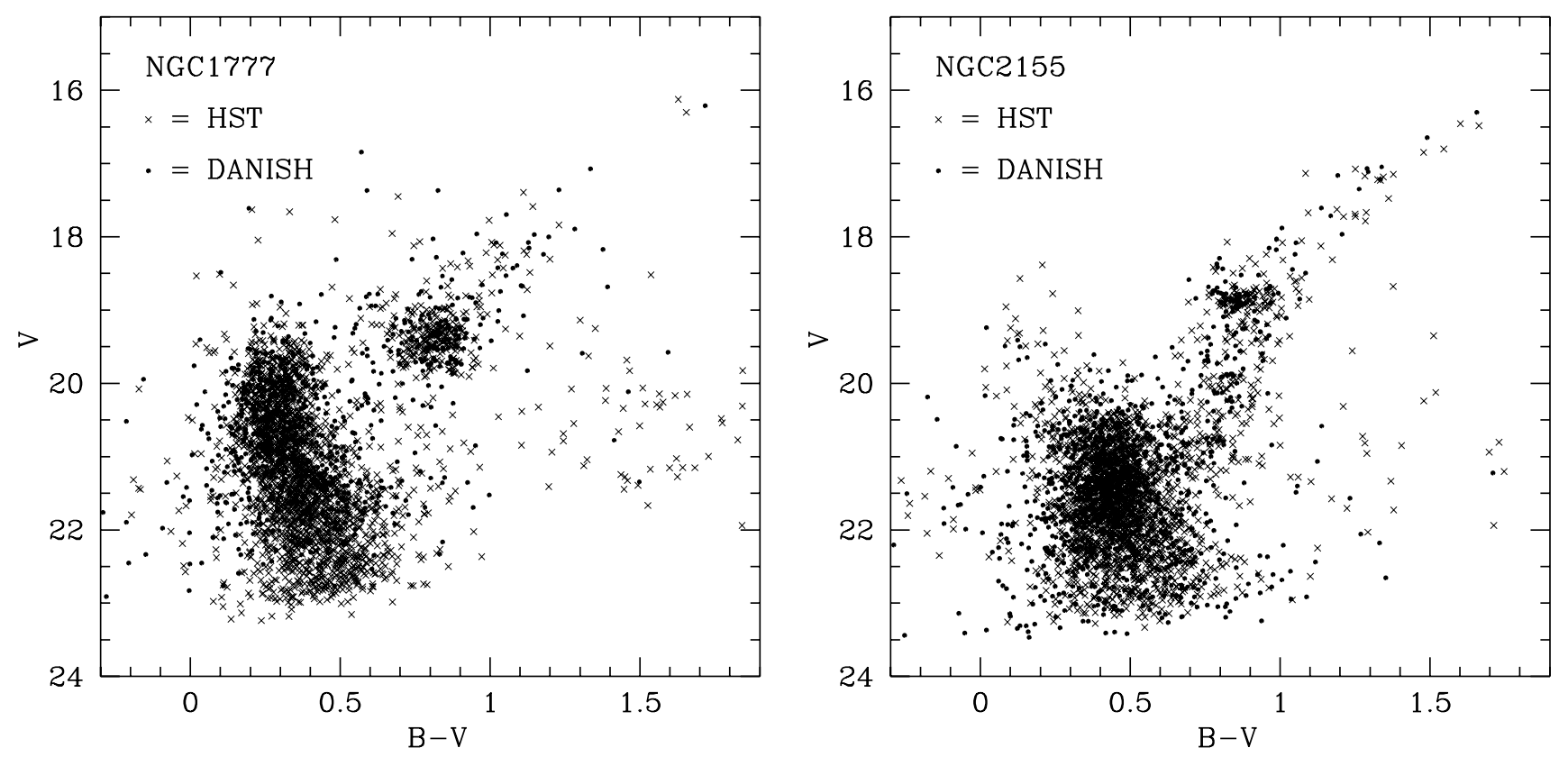

Fig. 4. Left panel: superposition of HST and Danish CM diagrams for the cluster NGC 1777; note that the present diagram does not include stars with $R<25$ arcsec. Right panel: the same for the cluster NGC 2155; note that our diagram does not include stars with $R<16$ arcsec.

resulted less than $0.01 \mathrm{mag})$. In Table 3 we summarise the adopted relations for calibration.

\subsection{Comparison with previous HST photometry}

LMC clusters NGC 1777 and NGC 2155 were already observed in the previous investigation, thus one can perform a comparison between HST and the present photometry. Left panel of Fig. 4 shows that the Danish CM diagram appears not worse than in the previous investigation, even though NGC 1777 with respect to the other clusters was observed in relatively bad seeing condition and with a shorter time exposure for deep frames. The obvious improvement of the HST diagram is to provide photometric measurements of stars located in the central region of the clusters and to reach a larger limiting $V$ magnitude, though by less than 1 mag. The CM diagrams of NGC 2155 presented in right panel of the same figure support quite similar considerations.

For the 436 stars of NGC 1777 in common (Figs. 5a, 5c), one finds a discrepancy of the order of $\langle\Delta V\rangle=\left\langle V_{\text {Danish }}-V_{\mathrm{HST}}\right\rangle \sim-0.04 \mathrm{mag}$, while colours appear in better agreement, with $\langle\Delta(B-V)\rangle=$ $<(B-V)_{\text {Danish }}-(B-V)_{\mathrm{HST}}>\sim 0.02 \mathrm{mag}$. Note that rather large number of stars with $<\Delta V\rangle<-0.4$ (i.e. stars of present photometry brighter than in the HST measurement) can be understood in terms of a blending effect in our photometry due to the worse seeing. In the case of NGC 2155, the same kind of comparison for 274 stars in common (Figs. 5b, 5d), discloses a small difference in $V$, of the order $\langle\Delta V\rangle \sim 0.03-0.04$ mag and a good agreement in colour $(<\Delta(B-V)>\sim-0.01 \mathrm{mag})$.
We conclude that Danish and HST investigations appear consistent within $0.1 \mathrm{mag}$.

\section{Colour-magnitude diagrams}

As an initial step in constructing the CMD for each cluster, we estimated the distance $R_{\mathrm{f}}$ from the cluster center (the point with the maximum stellar density) where the average cluster contribution becomes negligible with respect to the background stellar field. This step has been accomplished by evaluating the radial stellar distribution, defined as

$\rho(R)=\frac{\mathrm{d} N(R)}{2 \pi R \mathrm{~d} R}$

where $\mathrm{d} N(R)$ is the number of stars in the annulus of thickness $\mathrm{d} R$ at distance $R$ from the cluster center.

The Danish data are affected by substantial incompleteness in the crowded central region of clusters, so central values for $\rho(R)$ are not accurate; this would lead to a serious overestimate of the "core radius" $R_{\mathrm{c}}$ of clusters, defined as the radius for which the density in number of the measured stars is half of the central maximum. As an example, for NGC 2155 we would evaluate $R_{\mathrm{c}} \sim 45$ arcsec, against $R_{\mathrm{c}} \sim 13 \operatorname{arcsec}$ (Brocato et al. 2001) given by HST data. Even with such an overestimate, in our cluster sample we found $R_{\mathrm{c}}^{\max } \sim 50$ arcsec, in the case of IC 2146 and NGC 152. To be conservative, we decided to assume for all clusters $R_{f}=2 R_{\mathrm{c}}^{\max }=100$ arcsec. Beyond 100 arcsec from the center all the radial distribution are quite flattened, showing that only few cluster stars are still present. Thus stars at distance of $R>R_{\mathrm{f}}=100 \operatorname{arcsec}$ from the cluster center can be safely regarded as field stars. 


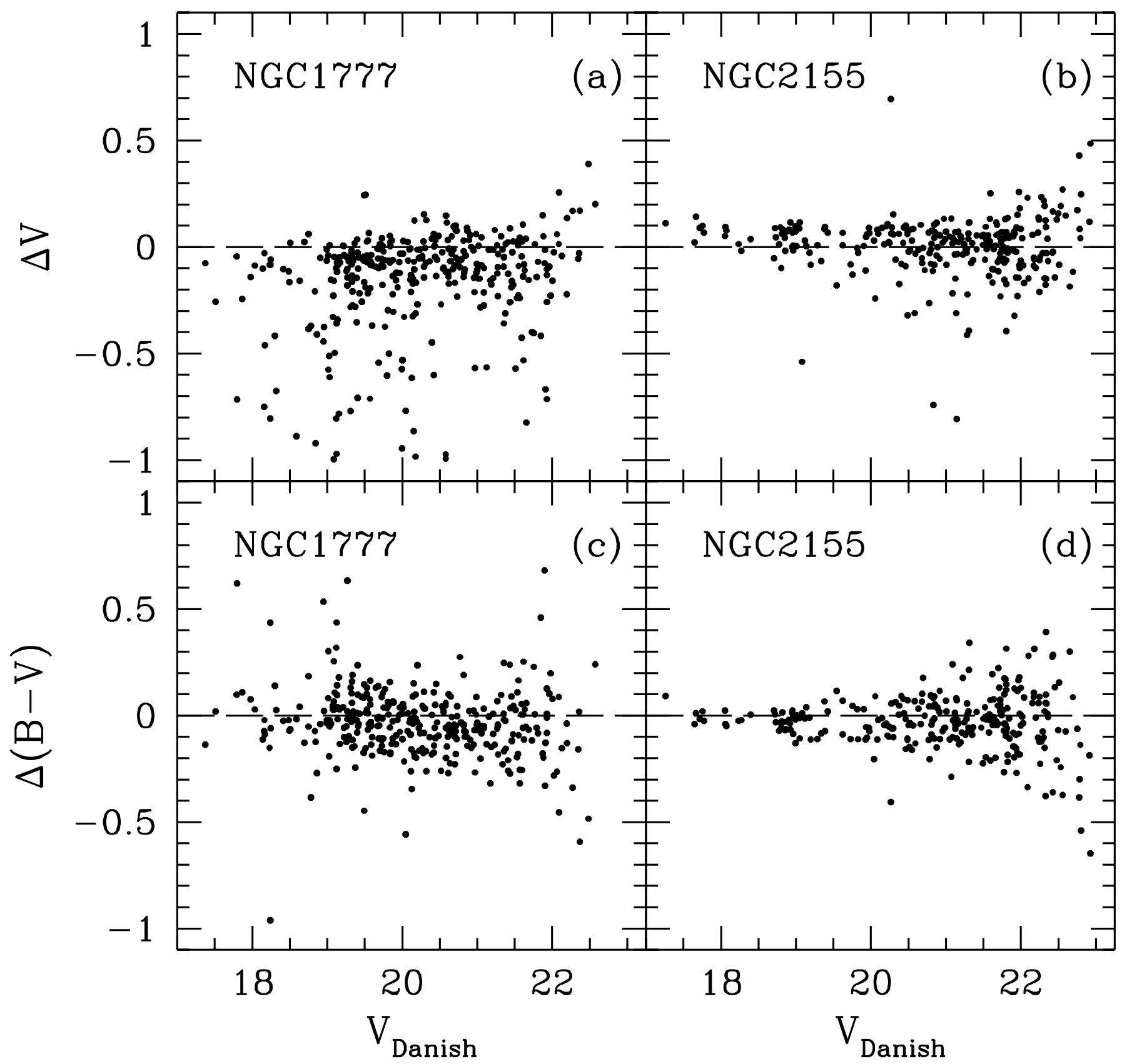

Fig. 5. Panels a) and c): comparison between present and HST photometry for NGC 1777. Panels b) and d): the same for NGC 2155.

In order to obtain the best CMD, for each cluster we tried to avoid including: i) field stars; ii) the regions of the cluster where the crowding is too much severe (only in the case of crowded clusters). To this aim, we considered CMDs for cluster stars in four different concentric annuli of increasing radii, being the outer annulus representative of the distance from the cluster center where the radial distribution merges into the surrounding field. In general the best CMD was obtained by using stars within the two inner annuli (see Fig. 7 and following). More details about this procedure can be found in Matteucci (2001).

All the photometric data are available as ASCII files via WEB (http://www.te.astro.it/pub/mcII/).

\section{1. $L M C$}

CCD images have been obtained for the LMC clusters NGC 1777, NGC 2155, IC 2146, KMHK264, KMHK265 and NGC 1902 (with NGC 1777 and NGC 2155 already observed in the previous investigation). Figure 6 shows the sky distribution of the clusters observed in both the present and the previous HST investigation. The right panel in the same figure provides the location of our sample in the integrated $(U-B),(B-V)$ diagram of 135 LMC clusters (Van den Bergh 1981), except KMHK264 and KMHK265 for which no integrated colours are available in literature. 

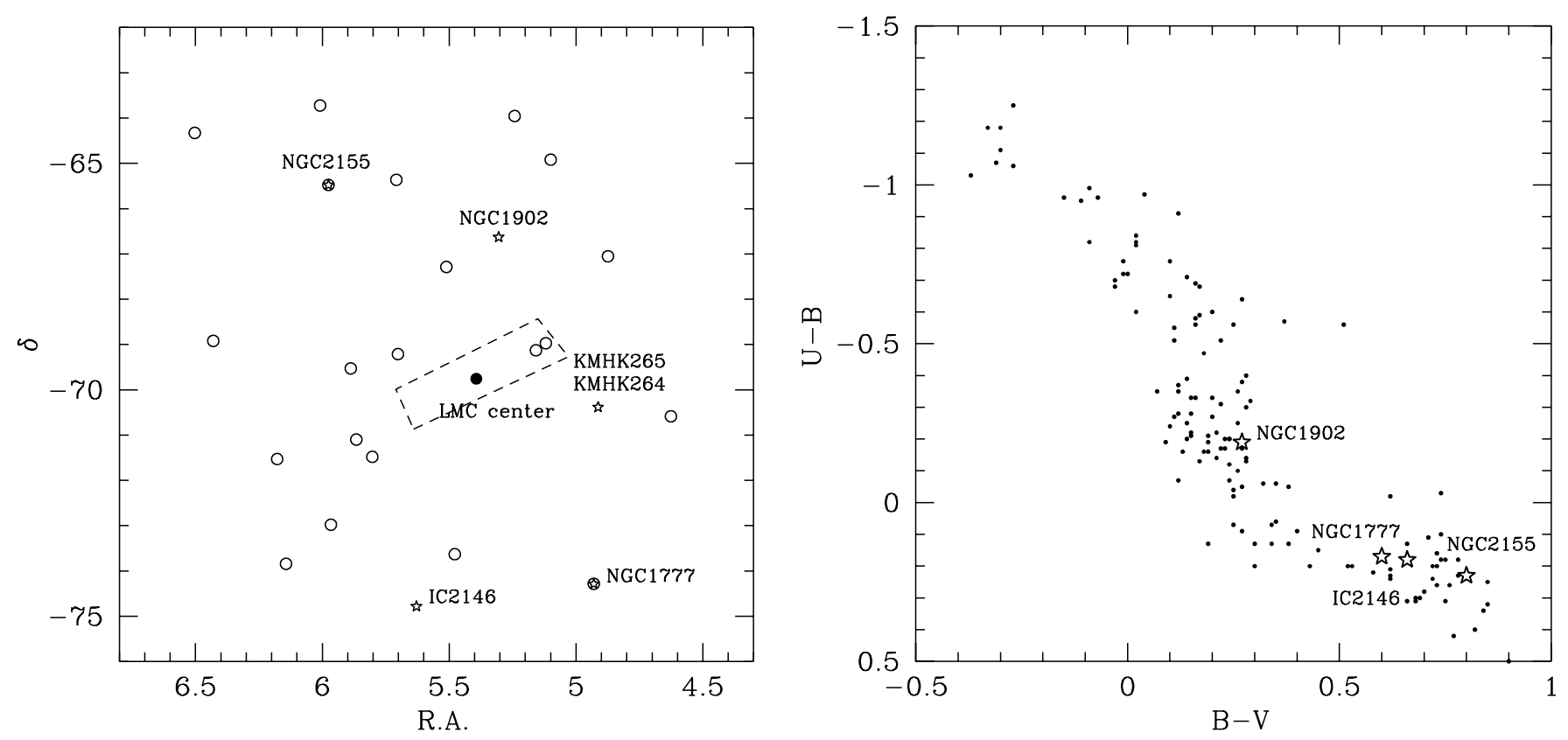

Fig. 6. Left panel: apparent spatial distribution of the investigated LMC clusters. Open circles indicate objects studied by Brocato et al. (2001), whereas dashed lines roughly delimitate LMC Bar ( $\delta$ and RA units are degree and hour, respectively). Right panel: the integrated properties of 4 LMC clusters investigated in this work are compared to a sample representative of the global cluster population of LMC; no data are available in literature for KMHK264 and KMHK265.

For each cluster, we present a figure divided in 4 panels with the following meaning: 1) an image, of a selected size of the CCD frame, showing the cluster with overimposed the annuli discussed before (top left); 2) the radial stellar distribution (top right); 3) the cluster CMD as obtained within the labelled distance from the center (bottom left); 4) the CM diagram for field stars $\left(R_{\mathrm{f}}>100\right.$ arcsec $)$ as obtained by randomly selecting a constant number $N_{\mathrm{f}}=$ 1950 of stars brighter than $V=21$ mag (bottom right). This choice will allow a significative comparison of field populations, by relying on similar sample of stars well above the limiting magnitude and not heavily affected by crowding.

Note also that in panel 4 (bottom right) the number $N_{\text {tot }}$ indicates the total number of field stars "measured" in Region 1 of CCD frame and not the number of "plotted" dots. In the same panel 4 , we also report the ridgelines of the two galactic globular clusters M 15 (Durrell \& Harris 1993) and 47 Tuc (Sosin et al. 1997) as boundary references of stellar populations with low and high values of metallicity (respectively $[\mathrm{Fe} / \mathrm{H}]=-2.25$ and -0.76 according to Harris 1996).

\subsubsection{NGC 1777}

A previous investigation on this cluster has been presented by Mateo \& Hodge (1985), relying on CCD images taken at the CTIO 4-m Telescope, reaching a limiting magnitude of about $V \sim 22 \mathrm{mag}$, as in our investigation. Comparison of stars in common (as well as of the CM diagrams), discloses a good agreement in the mean calibration, but with a better accuracy of present data.
The radial stellar distribution for NGC 1777 in Fig. 7 discloses that the contribution of cluster stars becomes negligible at $R \sim 70$ arcsec. After elimination of the stars in the inner annulus $(R<25$ arcsec), badly affected by crowding, the CMD (804 stars) of the cluster shows a main sequence (MS) terminating at $V \sim 19.5 \mathrm{mag}$ and a well defined red clump at the similar magnitude, with a slightly developed red giants branch (RGB) but without any clear evidence for a subgiants branch (SGB).

The CMD of the surrounding field presents some differences: there is marginal evidence for a population coeval with the cluster, but the field shows well developed RGB and SGB. Even though the field sample is more abundant by a factor 2.4 , this appears a real difference, therefore we conclude that the field includes a large population of stars older than the cluster one.

\subsubsection{NGC 2155}

Previous photometric studies of NGC 2155 have been presented by Sarajedini (1998) and by Brocato et al. (2001), both on the basis of HST snapshot. The great advantage of HST analysis has been the photometry of the inner cluster region: our diagram of stars with $R<16$ arcsec is indeed badly affected by crowding. The radial stellar distribution in Fig. 8 discloses that the numerical contribution of the cluster becomes negligible at $R \sim 80$ arcsec.

The cluster CMD (1085 stars) shows a MS terminating at $V \sim 20.5 \mathrm{mag}$, a red clump about 2 mag more luminous and sufficiently well defined SGB and RGB.

The CMD of the surrounding field presents again a component with similar features, but with parallel 

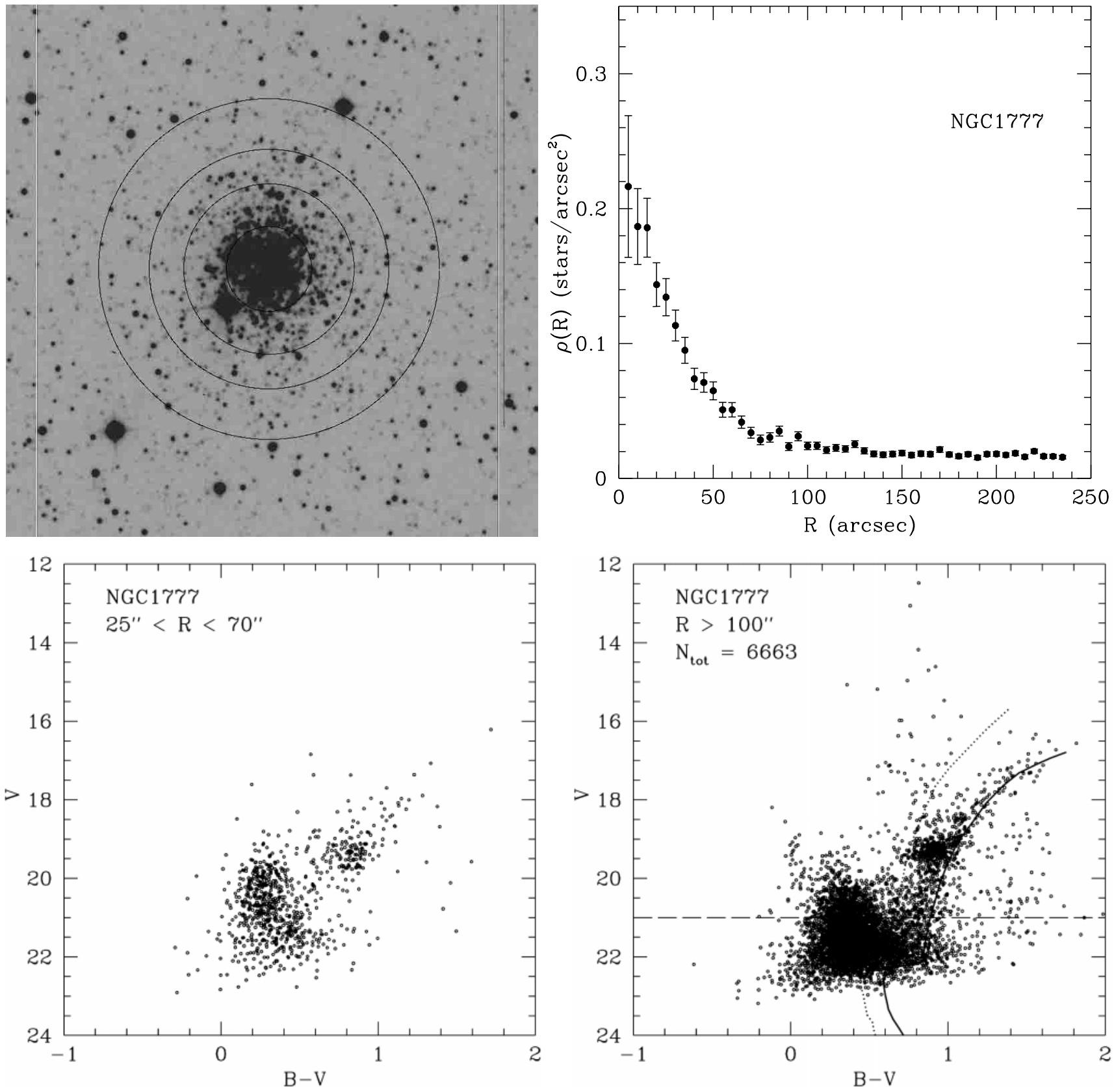

Fig. 7. In the left upper panel, the annuli investigated for NGC 1777 of radius $25^{\prime \prime}, 50^{\prime \prime}, 70^{\prime \prime}$ and $100^{\prime \prime}$ respectively; in the right upper panel, the radial stellar distribution around the NGC 1777 center. In the lower panels, the cluster and the field CM diagrams. Note that, we have reported on the diagram a sample of 6000 stars (4050 are fainter than $V=21$ mag); ridge lines of M 15 (dotted line) and 47 Tuc (solid line) clusters are overimposed (see Sect. 4 for details).

evidence for a substantial number of stars being younger than the cluster, with a MS reaching $V \sim 18.5$ mag and a red clump slightly fainter then the cluster one (see Girardi et al. 1998 for a discussion on the variation of the clump luminosity with stellar age).

\subsubsection{IC 2146}

There are no previous investigations on this cluster. The radial stellar distribution for IC 2146 in Fig. 9 discloses that the numerical contribution of the field becomes dominant around $R \sim 100$ arcsec, The CMD (2023 stars) of the cluster shows a well populated MS, reaching $V \sim$ $20.25 \mathrm{mag}$, and a well defined red clump at $V \sim 19.2 \mathrm{mag}$, with scarcely populated RGB and SGB.

The CMD of the surrounding field appears rather similar to that of the cluster, but with the evidence for an additional older population producing the well populated RGB below the clump of He burning stars.

\subsubsection{KMHK264 and KMHK265}

KMHK264 and KMHK265 are two small clusters observed in the same frame, near the older NGC 1754, this last 

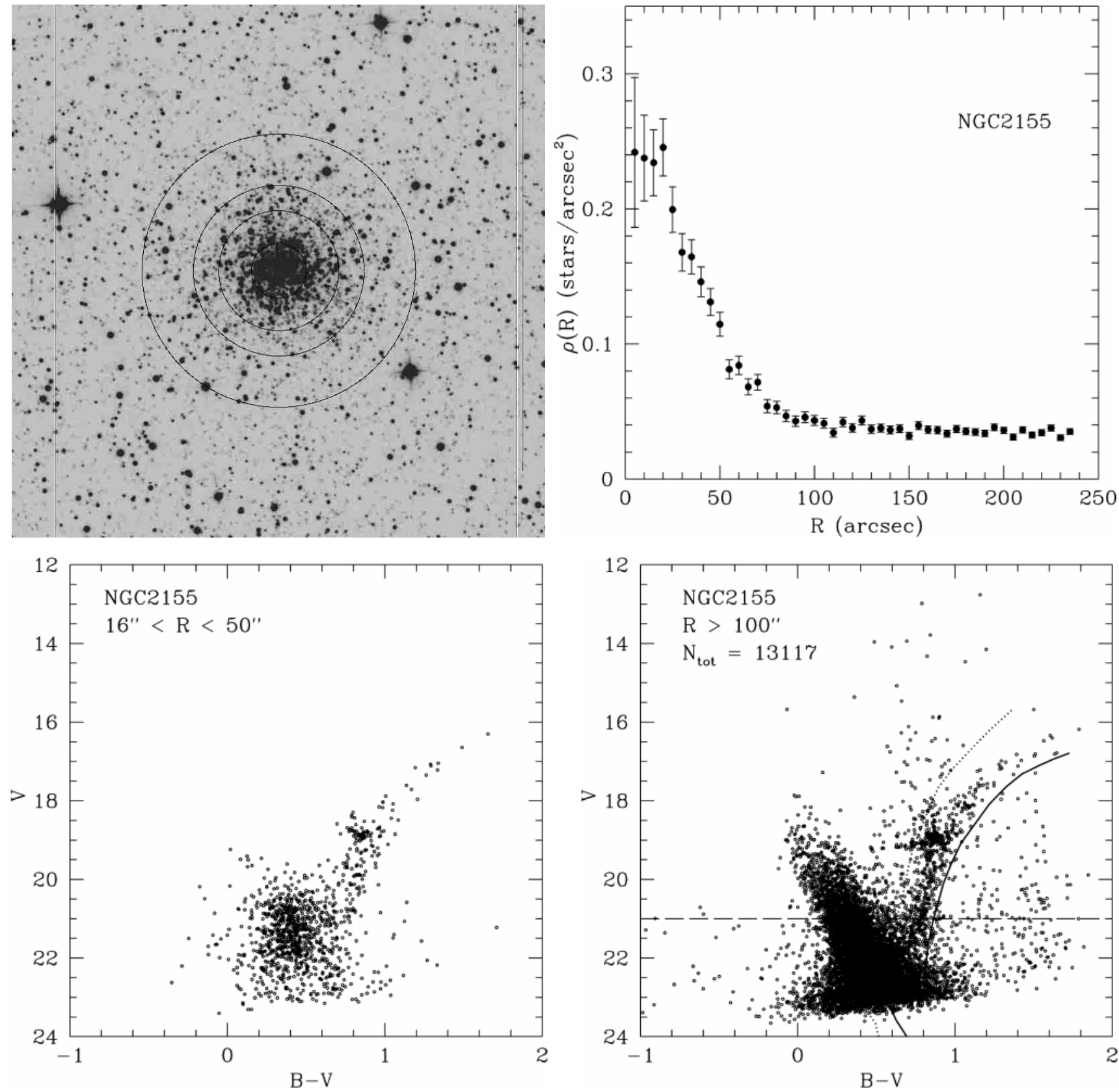

Fig. 8. In the left upper panel, the annuli investigated for NGC 2155 of radius $16^{\prime \prime}, 35^{\prime \prime}, 50^{\prime \prime}$ and $80^{\prime \prime}$ respectively; in the right upper panel, the radial stellar distribution around the NGC 2155 center. In the lower panels, the cluster and the field CM diagrams. Note that we have reported on the diagram a sample of 10000 stars (8050 are fainter than $V=21$ mag); ridge lines of M 15 (dotted line) and 47 Tuc (solid line) clusters are overimposed (see Sect. 4 for details).

being too crowded to obtain a reasonable CMD from present observations. Both clusters have no previous photometry.

The CMD (241 stars) of KMHK264 (Fig. 10) discloses a MS extending to $V \sim 17.5 \mathrm{mag}$, whereas KMHK265 CMD shows a MS up to $V \sim 16.5 \mathrm{mag}$, showing that both clusters have a relatively young age.

As for the field, we considered objects with $R^{(1)}>$ 85 arcsec from NGC 1754 center, $R^{(2)}>33$ arcsec from KMHK264 center and $R^{(3)}>41$ arcsec from KMHK265 center. The young population of the field appears rather similar to that of the clusters. It is also possible to find an older population identifiable in the well defined SGB, red clump and RGB.

\subsubsection{NGC 1902}

There are no previous investigations on this cluster. The radial stellar distribution for NGC 1902 in Fig. 12 shows that the numerical contribution of the cluster stars becomes dominant for $R<40$ arcsec.

The cluster CM diagram (440 stars) discloses the MS extending to $V \sim 17 \mathrm{mag}$, with some red giant stars possibly belonging to the cluster. 

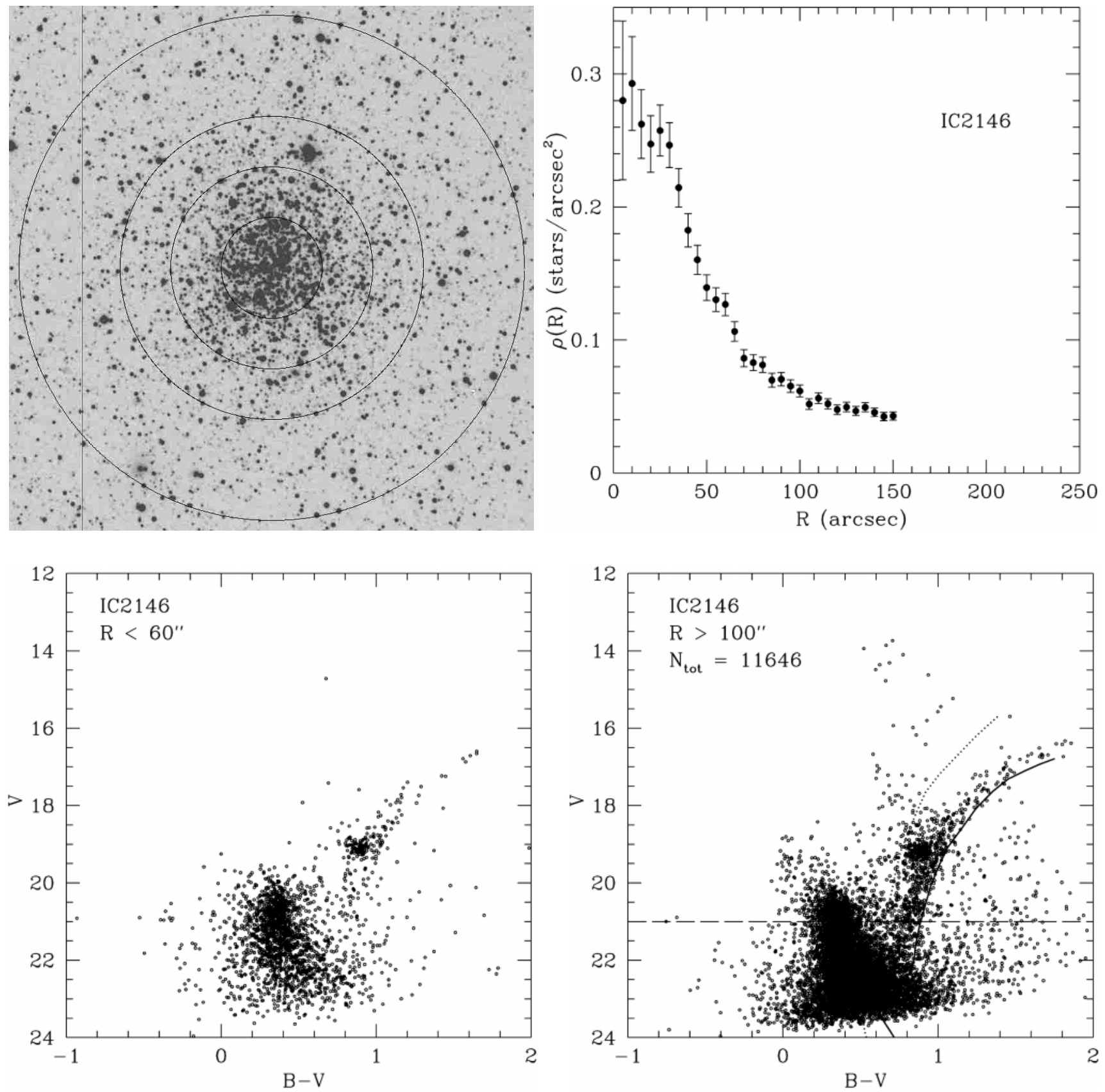

Fig. 9. In the left upper panel, the annuli investigated for IC 2146 of radius $30^{\prime \prime}, 60^{\prime \prime}, 90^{\prime \prime}$ and $150^{\prime \prime}$ respectively; in the right upper panel, the radial stellar distribution around the IC 2146 center. In the lower panels, the cluster and the field CM diagrams (we have reported 8050 stars fainter than $V=21 \mathrm{mag}$ ); ridge lines of M 15 (dotted line) and 47 Tuc (solid line) clusters are overimposed (see Sect. 4 for details).

In the surrounding field a similar young population is also recognisable by the well defined MS, together with an additional older component.

\section{2. $S M C$}

CCD images have been obtained for the SMC clusters L9, L13, L45, NGC 299, NGC 152, NGC 220, NGC 222, NGC 231, NGC 458 and NGC 643. The left panel of Fig. 13 shows the sky distribution of these objects; the open circle indicates Kron3, already investigated by Brocato et al. (2001). The $(U-B)$ and $(B-V)$ integrated colours of the SMC clusters under investigation (values are not available for L9, L13 and NGC 643) are reported in the right panel of the same figure, together with the data of 44 SMC clusters by Van den Bergh (1981).

The CM diagrams for fields again contain a constant number $N_{\mathrm{f}}=1950$ of stars brighter than $V=21$ mag except for NGC 643 region, where we found only 379 objects. 

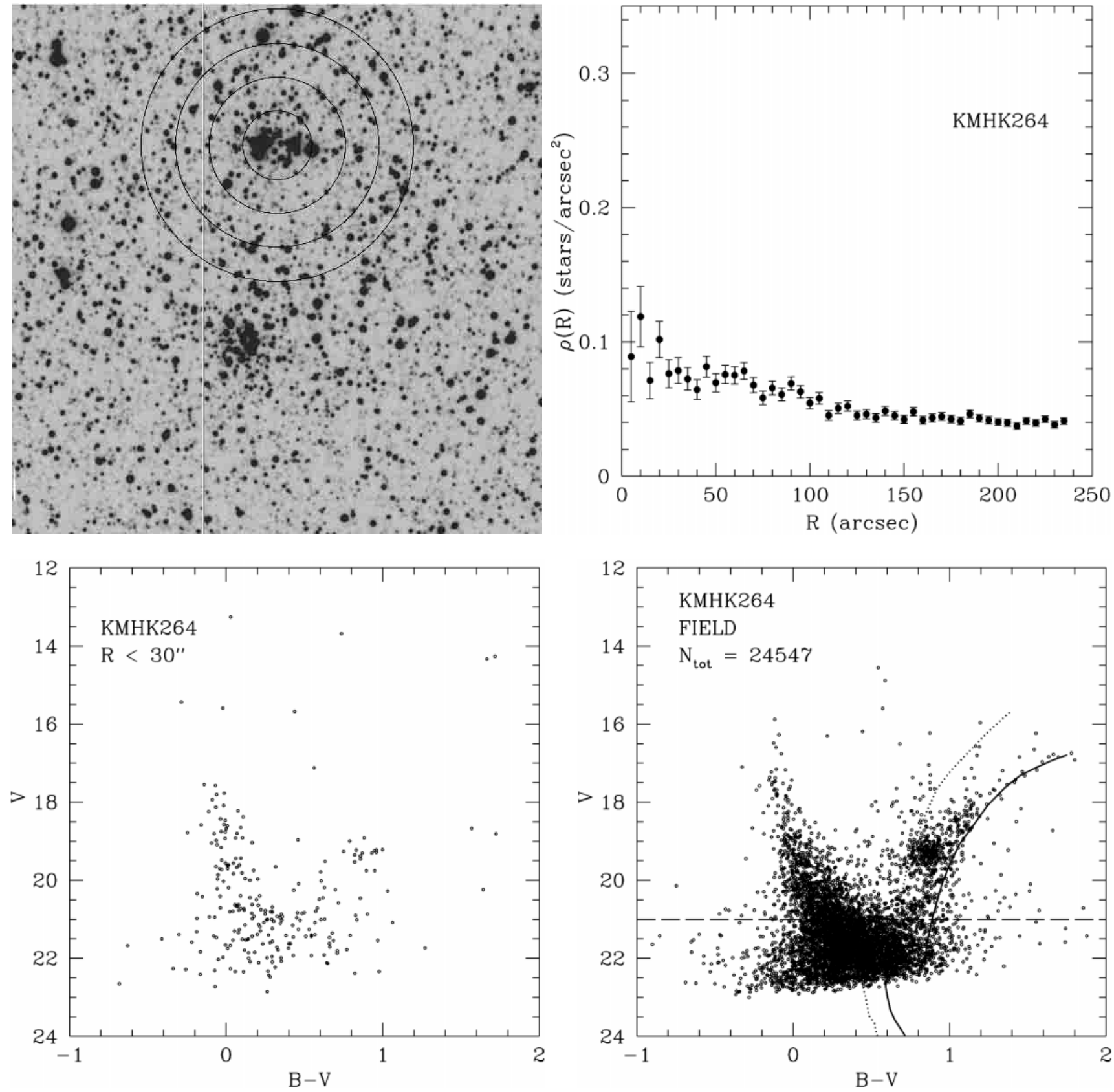

Fig. 10. In the left upper panel, the annuli division of KMHK264 region, of radius $15^{\prime \prime}, 30^{\prime \prime}, 45^{\prime \prime}$ and $60^{\prime \prime}$ respectively; in the right upper panel, the radial stellar distribution around the KMHK264 center. In the lower panels, the cluster and the field CM diagrams (we have reported 4050 stars fainter than $V=21 \mathrm{mag}$ ); ridge lines of M 15 (dotted line) and 47 Tuc (solid line) clusters are overimposed (see Sect. 4 for details).

\subsubsection{L9}

This is the first time that L9 has been investigated. The plot of radial stellar distribution in Fig. 14 reveals that the contribution of cluster stars becomes negligible at $R \sim 50$ arcsec. The CMD (374 stars) discloses the MS extending to $V \sim 20.25 \div 20.5 \mathrm{mag}$ and a red clump about 1 mag more luminous, with a slightly developed RGB and scarce evidence (if any) for SGB.

The surrounding field shows evidence for stars either much younger (with a MS reaching $V \sim 17.5 \mathrm{mag}$ ) or much older than the cluster, as revealed for the older population by the globular cluster-like RGB.

\subsection{2. $\mathrm{L} 13$}

This is a cluster so merged into the surrounding field that it is quite difficult to decide which star really belongs to L13. No previous investigations have been performed on it. The radial stellar distribution for L13 in Fig. 15 shows that the contribution of field stars becomes dominant at $R \sim 35$ arcsec. The cluster CMD (300 stars) discloses the 



Fig. 11. In the left upper panel, the annuli division of KMHK265 region, of radius $15^{\prime \prime}, 30^{\prime \prime}, 45^{\prime \prime}$ and $60^{\prime \prime}$ respectively; in the right upper panel, the radial stellar distribution around the KMHK265 center. In the lower panel, the cluster CM diagram.

MS extending to $V \sim 19.25$ mag and a group of helium burning stars.

Field CMD presents a quite old population, marked by the well developed RGB, with the additional contribution of a population reaching an age similar, if not smaller, to the age of cluster stars.

\subsubsection{L45 and NGC 299}

These clusters are present in the same field; both were not previously investigated. Radial distributions (Figs. 16 and 17) reveal that the numerical contribution of the field becomes dominant at $R \sim 30$ arcsec. L45 CMD (334 stars) shows a MS extending to $V \sim 17 \mathrm{mag}$, with possibly some He burning giants. NGC299 CMD (271 stars) discloses an even more extended MS (to $V \sim 14.5 \mathrm{mag}$ ) and three probable red He burning giants at $[(B-V), V] \sim$ $[1.6,13.5] \mathrm{mag}$.

We considered all objects with $R^{(1)}>76$ arcsec from L45 center and $R^{(2)}>65$ arcsec from NGC 299 center as stars belonging to stellar field. As shown in Fig. 16, the field presents again a large range of ages, with stars possibly as young as in the clusters but also with a globular cluster-like population. 

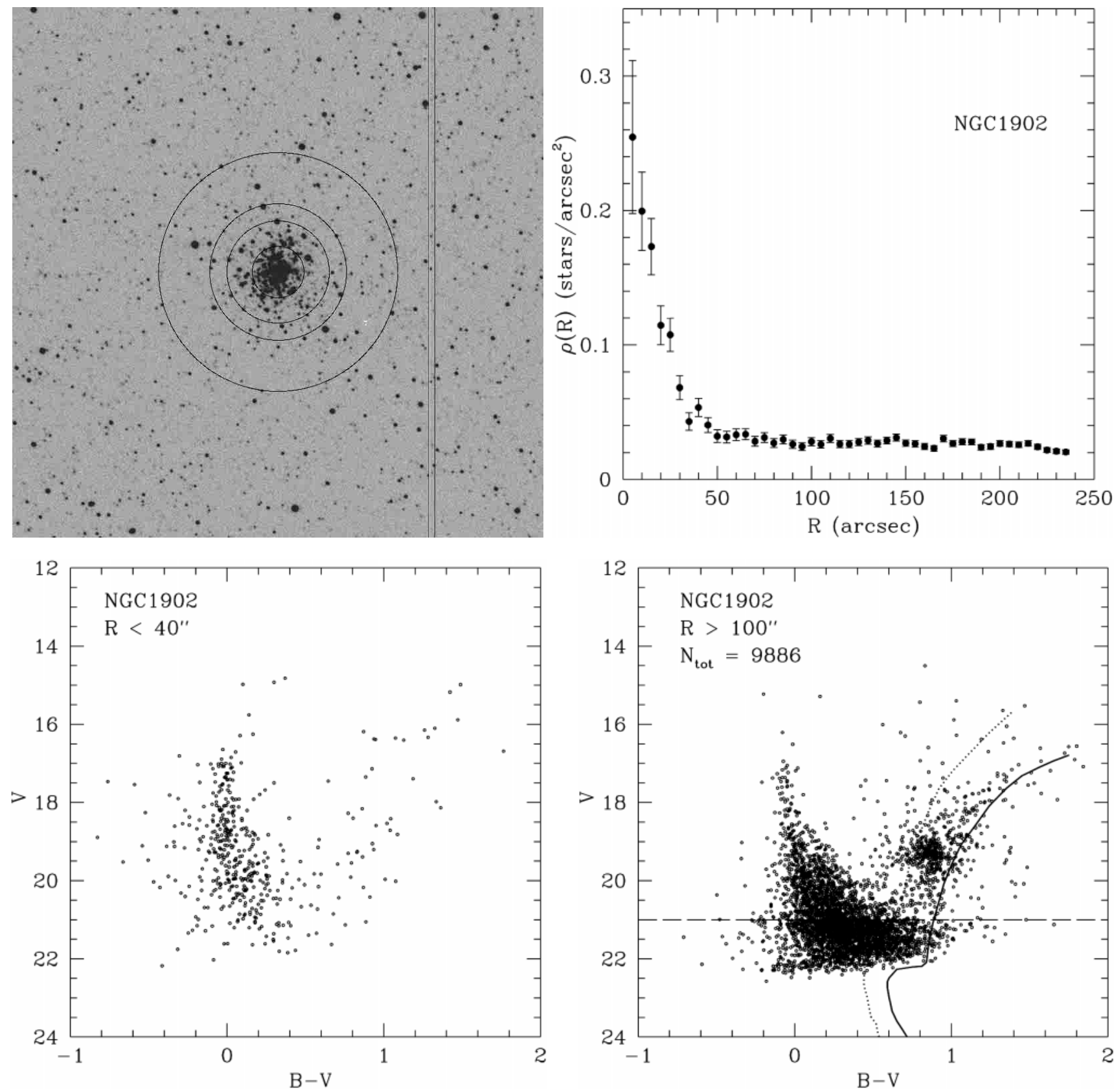

Fig. 12. In the left upper panel, the annuli division of NGC 1902 region, of radius $15^{\prime \prime}, 30^{\prime \prime}, 40^{\prime \prime}$ and $70^{\prime \prime}$ respectively; in the right upper panel, the radial stellar distribution around the NGC 1902 center. In the lower panels, the cluster and the field CM diagrams (we have reported 2450 stars fainter than $V=21 \mathrm{mag}$ ); ridge lines of M 15 (dotted line) and 47 Tuc (solid line) clusters are overimposed (see Sect. 4 for details).

\subsubsection{NGC 152}

A previous investigation of this cluster has been presented by Hodge (1981), as based on photographic plates taken at the CTIO 4-m Telescope, and by Rich et al. (2000), as based on HST snapshot of the central region of the cluster. Radial stellar distribution in Fig. 18 shows that cluster contribution tends to disappear at $R \sim 90$ arcsec. The cluster CMD (1862 stars) discloses a well defined MS reaching $V \sim 20.25 \div 20.5 \mathrm{mag}$, a red clump about $1 \mathrm{mag}$ more luminous and a RGB, with scarce evidence of a SGB.
Field stars have ages from much older than to similar to cluster stars, with marginal evidence for an even younger population with a MS extending to $V \sim 17.5 \mathrm{mag}$.

\subsubsection{NGC 220, NGC 222 and NGC 231}

Also these clusters are present in the same frame. Previous CMD for these clusters are available in the web site of $\mathrm{OGLE}^{1}$, as based on CCD observations at the 1.3-m

\footnotetext{
1 http://sirius.astrouw.edu.pl/ ogle/
} 

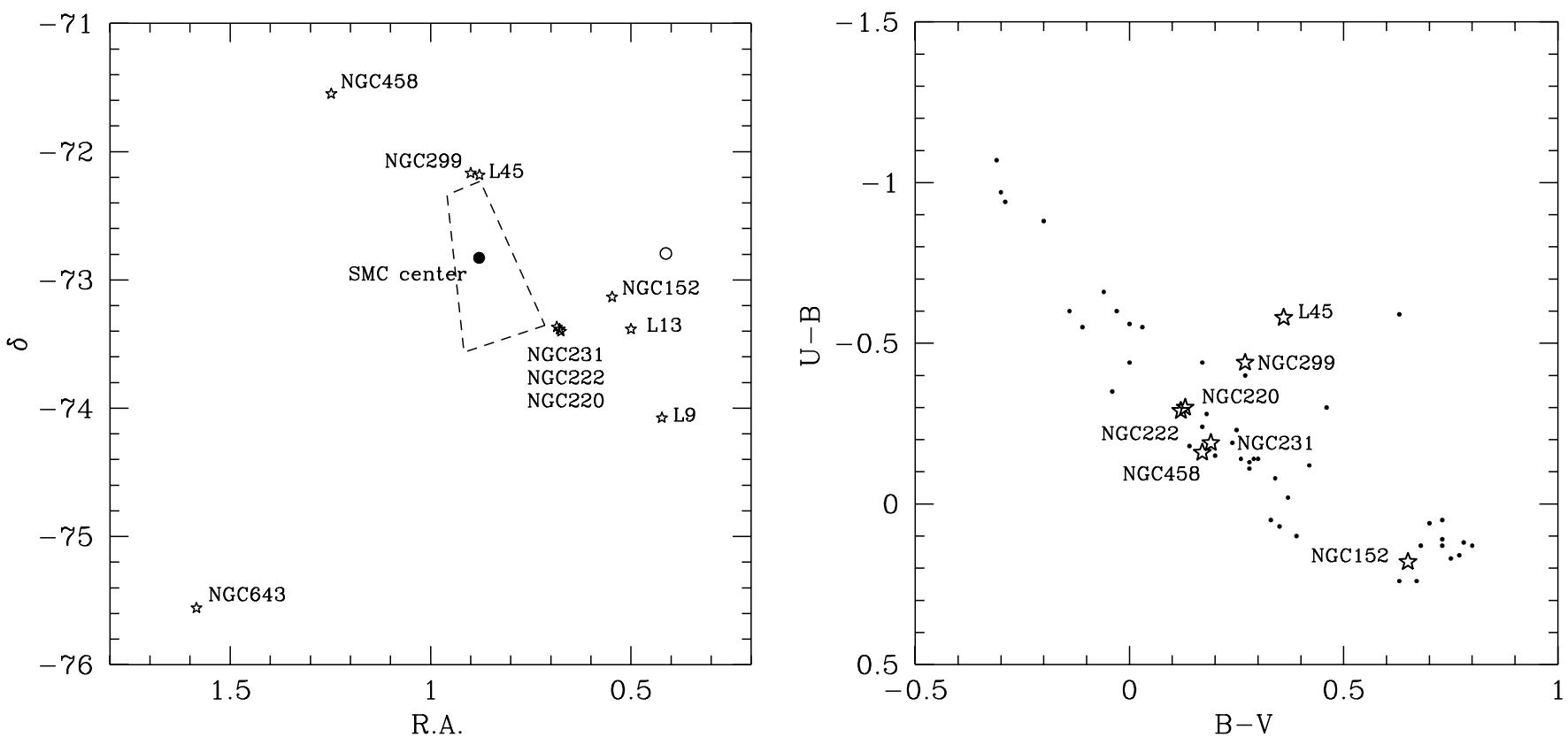

Fig. 13. Left panel: apparent spatial distribution of the investigated SMC clusters. The open circle indicates Kron3, whereas dashed lines roughly delimitate SMC Bar ( $\delta$ and RA units are degree and hour, respectively). Right panel: the integrated properties of $7 \mathrm{SMC}$ clusters investigated in this work are compared to a sample representative of the global cluster population of SMC; no data are available in literature for L9, L13 and NGC 643.

Warsaw Telescope at Las Campanas, Chile. A comparison with our CM diagrams discloses a good photometric agreement. Note also that our CMDs extend to stars 1 mag fainter.

Radial stellar distributions in Figs. 19-21 shows that the cluster contribution tends to disappear at $R \sim$ 35 arcsec in all cases. NGC 220, NGC 222 and NGC 231 CM diagrams (511, 361, 449 stars respectively) are quite similar, all disclose a MS extending to $V \sim 16.5$ mag with some core helium burning stars (see Figs. 19-21).

Field stars show again a large range of ages, starting from much older than or similar to cluster stars.

\subsubsection{NGC 458}

A pioneering investigation on this cluster has been presented by Arp (1959) on the basis of photographic plates. The radial stellar distribution for NGC 458 in Fig. 22 shows that the contribution of the field becomes dominant at $R \sim 60$ arcsec. The cluster CM diagram (1288 stars) discloses a well defined MS extending to $V \sim 17.0 \mathrm{mag}$ and more than 30 evolved star forming a quite developed "blue loop".

Field CMD discloses a similar and well populated young component, together with an older one.

\subsubsection{NGC 643}

There are no previous investigations on this cluster, which is located far from SMC center. The radial stellar distribution for NGC 643 in Fig. 23 shows that the numerical contribution of the cluster becomes negligible at
$R \sim 70$ arcsec, with a very small field density. The cluster CM diagram (1127 stars) discloses a well defined MS extending to $V \sim 19.5$ and a red clump at $[(B-V), V] \sim$ $[0.8,19]$ mag, with a poorly developed RGB.

The surrounding field is poorly populated, and the CMD discloses a MS reaching only $V \sim 20.5$, without evidence of younger stellar components.

\section{Cluster and field stellar populations}

Even though this paper is mainly devoted to the presentation of observational results, the CM diagrams presented in the previous section deserve some further comments. As a first point, let us summarise in Table 4 selected quantities for the studied stellar clusters, giving for each cluster the SWB class (Searle et al. 1980), the sky region used for producing the CM diagram, the number of stars $\left(N_{\text {star }}\right)$ in the diagram, the estimated $V$ magnitude of the upper MS termination $\left(V_{\mathrm{TO}}\right)$. The last column gives an estimate of the cluster ages as obtained from the $M_{V}^{\mathrm{TO}}$ age relation (Castellani et al., in preparation), assuming $Z=0.008,(m-M)_{V}=18.6$ for LMC and $Z=0.004$, $(m-M)_{V}=19.0$ for SMC. Because of the observational uncertainties in $V_{\mathrm{TO}}$ and lack of firm evaluations on the cluster metallicities in Table 4 we report just an indication of the range of ages which appears suitable for the various clusters. As expected from the SWB classification, one finds that clusters in both Clouds span a wide range of ages. To further discuss the comparison with theoretical predictions, as an example we show in Fig. 24 the stellar isochrones for the two extreme ages in Table 4 as overlapped on the CM diagram of stars in the LMC cluster 

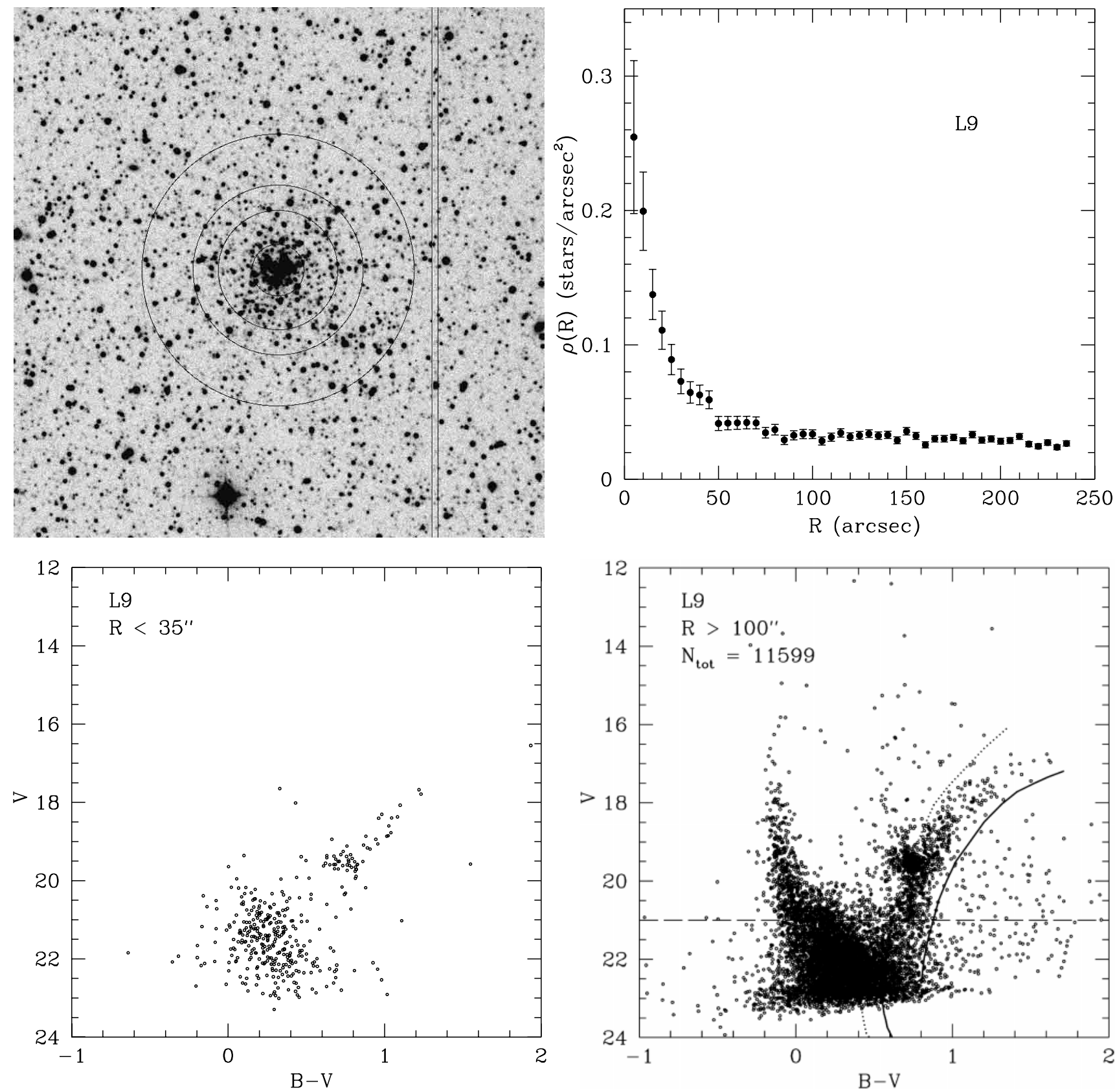

Fig. 14. In the left upper panel, the annuli division of L9 region, of radius $15^{\prime \prime}, 35^{\prime \prime}, 50^{\prime \prime}$ and $80^{\prime \prime}$ respectively; in the right upper panel, the radial stellar distribution around the L9 center. In the lower panels, the cluster and the field CM diagrams (we have reported 5550 stars fainter than $V=21 \mathrm{mag}$ ); ridge lines of M 15 (dotted line) and 47 Tuc (solid line) clusters are overimposed (see Sect. 4 for details).

IC 2146. One finds that, for the given chemical composition, the two isochrones indeed reasonably encompass the observed distribution of MS stars. Note that theoretical colours for red giants are freely governed by the assumptions on the efficiency of surface convection and the agreement between theory and observation is only marginally significant. This is not the case for luminosities, which keep being a firm theoretical prediction; the reasonable agreement between predicted and observed luminosities of the clumping He-burning red giants can be thus regarded as comforting evidence.
As for the surrounding fields, one finds in all cases evidence of an old globular cluster-like population, with an age of several billion years, as indicated by the occurrence of well-developed RGBs. A simple inspection of the CM diagrams does not reveal clear differences in the slope of these RGBs, either within the single Clouds, or between the two Clouds, excluding the occurrence of large variations in the metallicity of these earlier populations. However, comparison with ridge-lines for the two selected galactic globular clusters (M 15 and 47 Tuc), as presented in panel 4 of all figures for the given distance 

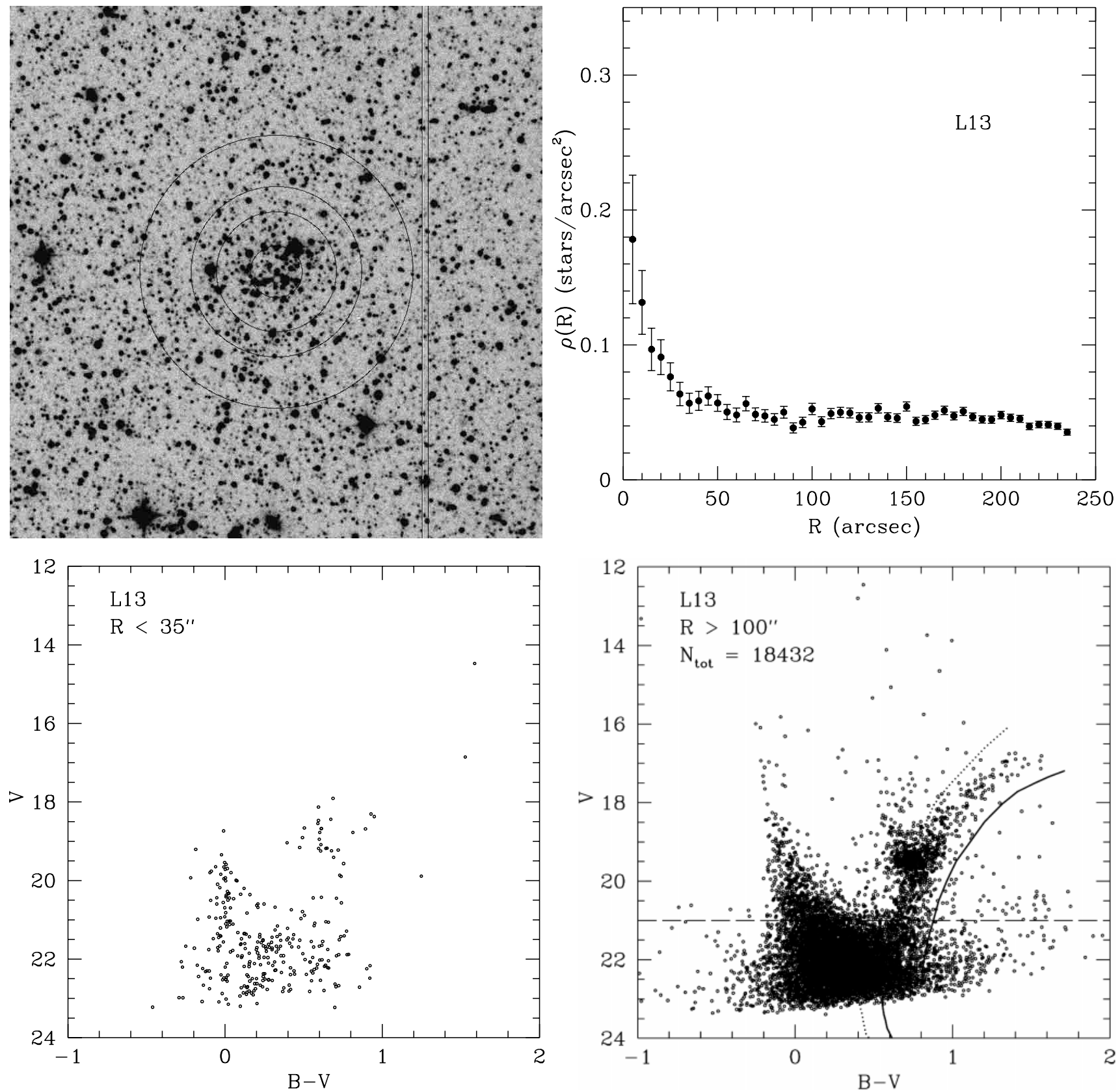

Fig. 15. In the left upper panel, the annuli division of L13 region, of radius $15^{\prime \prime}, 35^{\prime \prime}, 50^{\prime \prime}$ and $80^{\prime \prime}$ respectively; in the right upper panel, the radial stellar distribution around the L13 center. In the lower panels, the cluster and the field CM diagrams (we have reported 7450 stars fainter than $V=21 \mathrm{mag}$ ); ridge lines of M 15 (dotted line) and 47 Tuc (solid line) clusters are overimposed (see Sect. 4 for details).

moduli and adopting mean foreground reddening values from Schlegel et al. (1998) $E(B-V)=0.075$ and 0.037 for LMC and SMC respectively (except in the cases of NGC 2155 and NGC 643 fields, for which Schlegel et al. values are 0.051 and 0.068 respectively), reveals several relevant features. One finds that the metallicity of LMC old field stars appears a bit lower than stars in 47 Tuc, i.e., lower than $[\mathrm{Fe} / \mathrm{H}] \sim-0.76$. Moreover, one finds that the old population in SMC appears even more metal-poor, roughly between the metallicities of M15 and 47 Tuc, i.e.
$-2.25<[\mathrm{Fe} / \mathrm{H}]<-0.76$ (Harris 1996). One finds that reasonable variations in the adopted distance moduli and/or reddening cannot affect such a conclusion which, in turn, is not surprising, since it was already found (e.g. Olszewsky et al. 1996 and references therein) that field stars in both Clouds can be much less metallic than the nominal adopted metallicities (see e.g. Westerlund 1997, who reports $[\mathrm{Fe} / \mathrm{H}]_{\mathrm{LMC}}=-0.30 \pm 0.2$ and $[\mathrm{Fe} / \mathrm{H}]_{\mathrm{SMC}}=-0.65 \pm$ 0.2 for cool stars in the fields of the two galaxies). As a further support to this occurrence, Dolphin et al. (2001) 

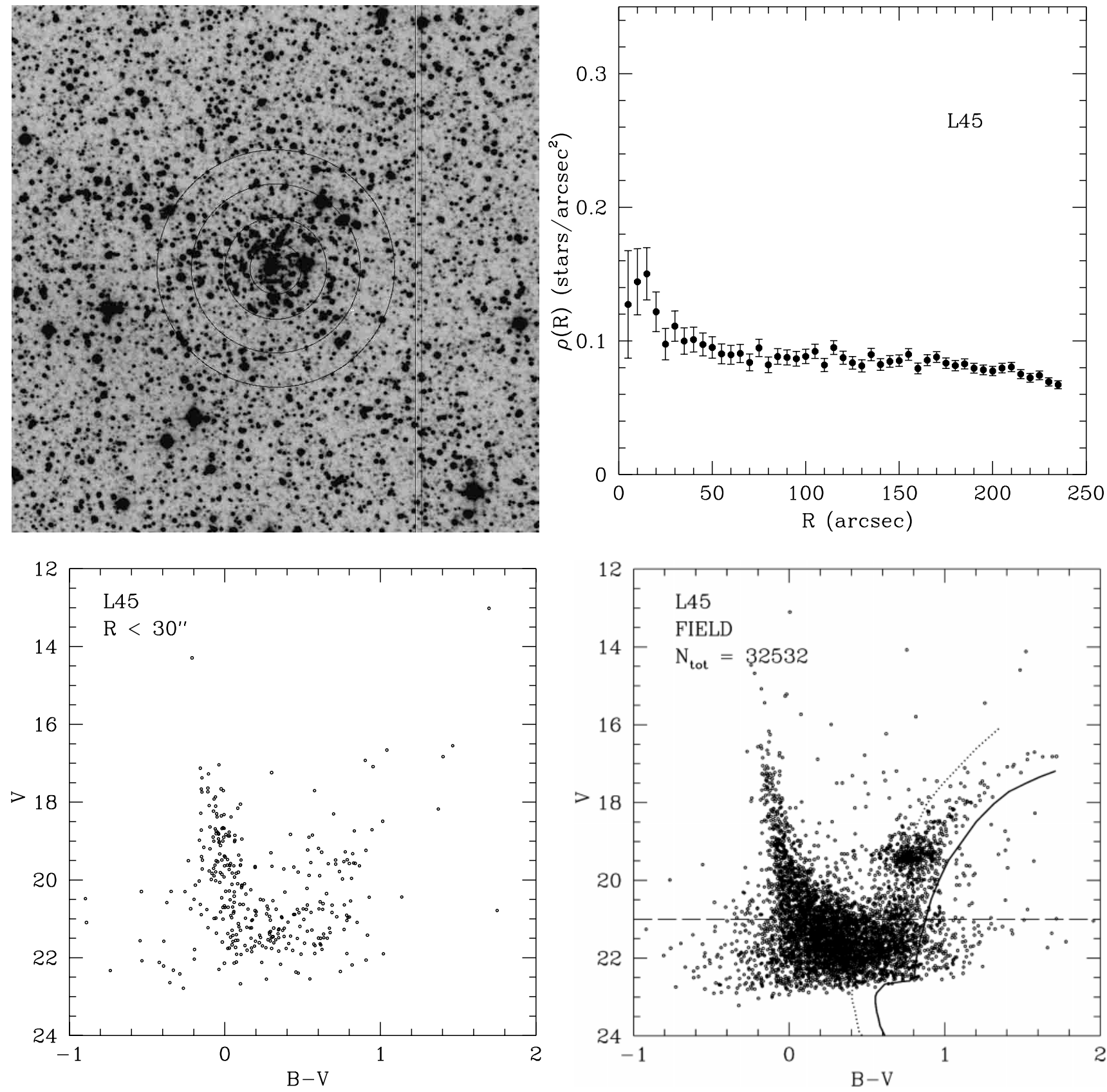

Fig. 16. In the left upper panel, the annuli division of L45 region, of radius $15^{\prime \prime}, 30^{\prime \prime}, 50^{\prime \prime}$ and $70^{\prime \prime}$ respectively; in the right upper panel, the radial stellar distribution around the L45 center. In the lower panels, the cluster and the field CM diagrams (we have reported 2950 stars fainter than $V=21 \mathrm{mag}$ ); ridge lines of M 15 (dotted line) and 47 Tuc (solid line) clusters are overimposed (see Sect. 4 for details).

have recently presented observational evidence for a metal poor $([\mathrm{Fe} / \mathrm{H}] \sim-1.3 \pm 0.3)$ population in the SMC halo region in the field of NGC 121.

In all fields one finds evidence of additional younger populations, but with the last episode of star formation placed at various ages. Not surprisingly, in all cases the age of the cluster appear within the range of ages covered by the surrounding field, sometimes in correspondence of the last episode of star formation (IC 2146), sometimes much earlier (e.g., L9). Only NGC 643 seems to be younger than the surrounding population, but we tend to attribute such peculiarity to the very low statistical significance of the field sample.

To go deeper in this scenario, let us discuss separately the fields of the two Clouds.

\subsection{LMC fields}

Stellar population studies, as based on CMD investigations, have already been extensively carried out with the aim of constraining the LMC star-formation history. In particular, one of the most interesting issues under 



Fig. 17. In the left upper panel, the annuli division of NGC 299 region, of radius $15^{\prime \prime}, 25^{\prime \prime}, 40^{\prime \prime}$ and $70^{\prime \prime}$ respectively; in the right upper panel, the radial stellar distribution around the NGC 299 center. In the lower panel, the cluster CM diagram.

discussion is whether common star-formation events involved the entire LMC or different regions of the Cloud experienced different star-formation histories. Bertelli et al. (1992), Vallenari et al. (1996a) and Vallenari et al. (1996b), on the basis of CCD ground observations of fields out of the Bar, found evidence that star-formation activity is correlated with position in the LMC. In the vicinity of the LMC main body, a significant young population component is present with an age of 0.06-0.1 Gyr; this age seems to increase to $0.4-0.5$ Gyr at $4^{\circ}$ from the LMC center and to more than $1 \mathrm{Gyr}$ beyond $5^{\circ}$; in a western outer field young stars seem to be completely absent.
Westerlund et al. (1995) investigated a region in the NW $\left(\sim 4^{\circ}\right.$ from the center) and one in the SW $\left(\sim 5^{\circ}\right)$, finding a young component $(0.2-0.6 \mathrm{Gyr})$ present only in the first zone; they have also found similar characteristics between fields and included clusters. Westerlund et al. (1998) studied three northeastern fields whose youngest population appears to follow an analogous trend: the inner field shows a very young component that is completely absent in the outer one. Santos et al. (1999) investigated 14 fields covering the central distance range $4-8 \mathrm{kpc}$ and confirmed the previous general behaviour. Geha et al. (1998), on the basis of HST deep observations, studied two fields in the 

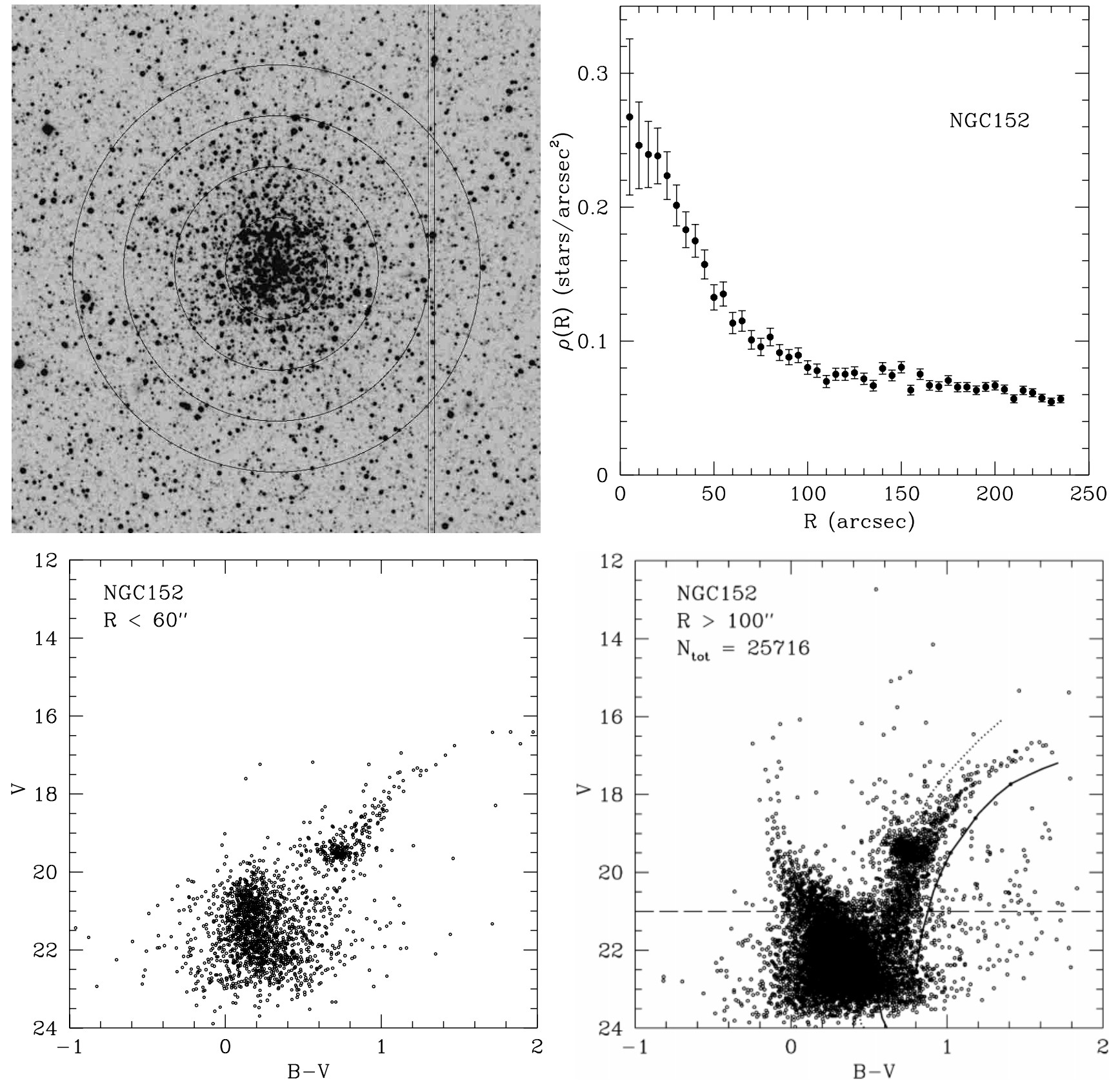

Fig. 18. In the left upper panel, the annuli division of NGC 152 region, of radius $30^{\prime \prime}, 60^{\prime \prime}, 90^{\prime \prime}$ and $120^{\prime \prime}$ respectively; in the right upper panel, the radial stellar distribution around the NGC 152 center. In the lower panels, the cluster and the field CM diagrams (we have reported 7050 stars fainter than $V=21 \mathrm{mag}$ ); ridge lines of M 15 (dotted line) and 47 Tuc (solid line) clusters are overimposed (see Sect. 4 for details).

NW region and one in the NE and found that they contain statistically indistinguishable stellar populations, in apparent contrast to the other quoted works.

To approach such a scenario in a more quantitative way, we made use of our field samples (cf. Sect. 3.1), dividing the CMDs for $V<21$ mag into two regions separating the MS from evolved red stars and counting the objects in each zone. Figure 25 gives an example of this procedure in the case of the KMHK264 field, while Table 5 reports star counts for LMC fields, where $N_{B}, N_{R}$ indicate the number of MS and evolved red stars, respectively. We can now connect these results with the field positions in the LMC.
More internal fields (KMHK264 and NGC 1902) present greater $N_{B}$ and, correspondingly, a more extended MS (see also Figs. 10 and 12); southern fields (NGC 1777 and IC 2146), instead, present greater $N_{R}$ and lack a young population component (see also Figs. 7 and 9): this evidence appears in good agreement with previous suggestions. The case of NGC 2155 field merits a particular remark: it presents indeed a large $N_{B}$ as more internal fields, but the MS does not extend to high luminosities, so the field has an abundant component younger than cluster stars, but not as young as in the fields around KMHK264 or NGC 1902. This evidence is in agreement 

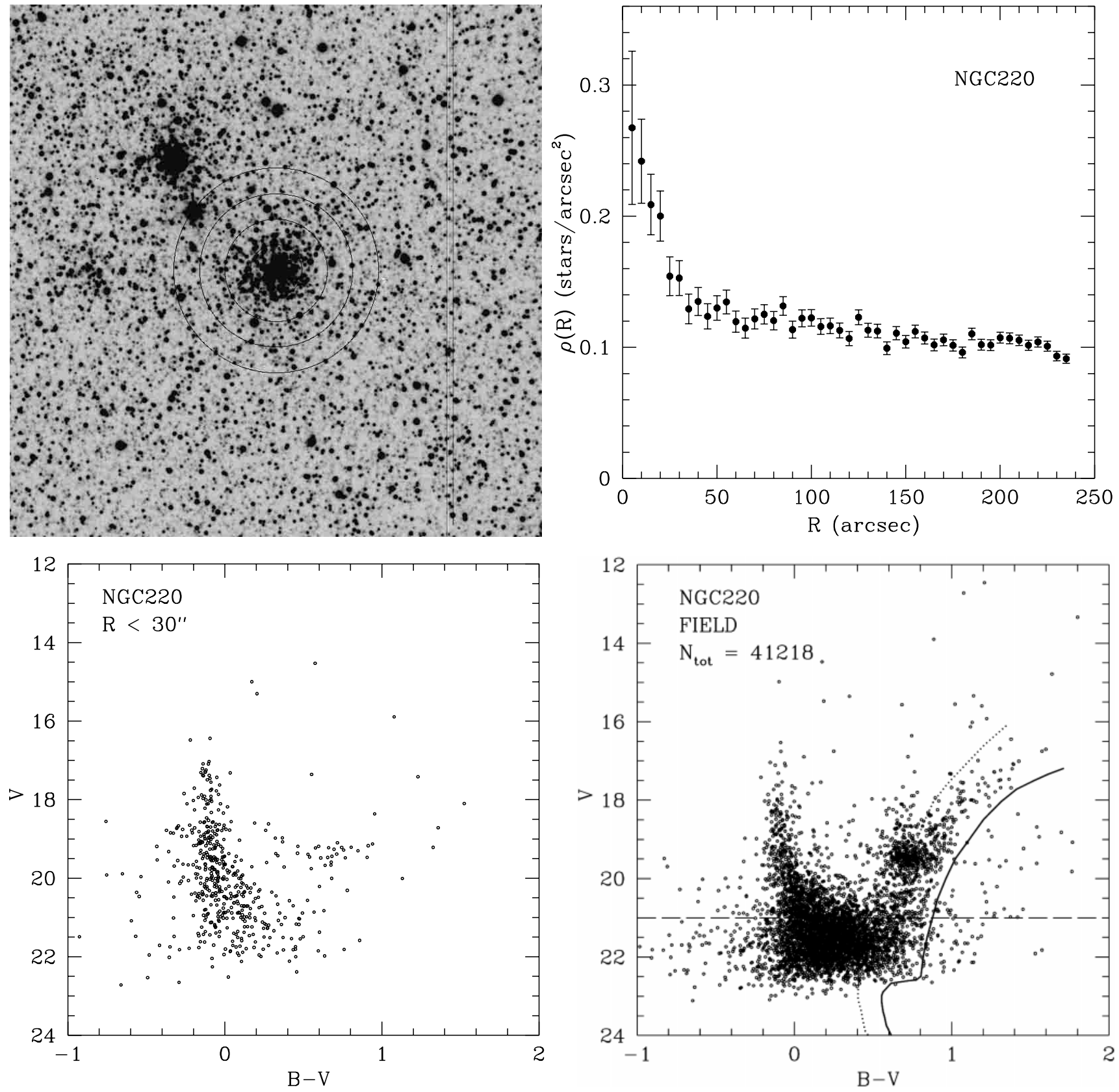

Fig. 19. In the left upper panel, the annuli investigated for NGC 220 , of radius $15^{\prime \prime}, 30^{\prime \prime}, 45^{\prime \prime}$ and $60^{\prime \prime}$ respectively; in the right upper panel, the radial stellar distribution around the NGC 220 center. In the lower panels, the cluster and the field CM diagrams (we have reported 2950 stars fainter than $V=21 \mathrm{mag}$ ); ridge lines of M 15 (dotted line) and 47 Tuc (solid line) clusters are overimposed (see Sect. 4 for details).

with Bertelli et al. (1992), which studied a smaller area near this field showing the same characteristic.

\subsection{SMC fields}

With respect to the Large Cloud, the field of the SMC and the Cloud formation history were less investigated. The most extensive work (Gardiner \& Hatzidimitriou 1992) has been the search for main-sequence stars younger than 2 Gyr in an area covering six UK Schmidt Telescope survey fields, virtually corresponding to the entire SMC outer region. These authors concluded that such MS objects are absent in the NW, while the younger population is considerably distributed over a large part of the eastern (the SMC "Wing") and southern areas, with a rapidly increasing concentration with decreasing distance from the optical center. A similar distribution has been noted for SMC clusters (Van den Bergh 1991), younger clusters being more concentrated towards the SMC Bar and the older ones more dispersed. Our sample allows us to extend the investigation towards more internal regions, not covered by the Gardiner \& Hatzidimitriou (1992) study.

Following the same procedure used for the LMC fields, we have performed star counts on MS and evolved regions 

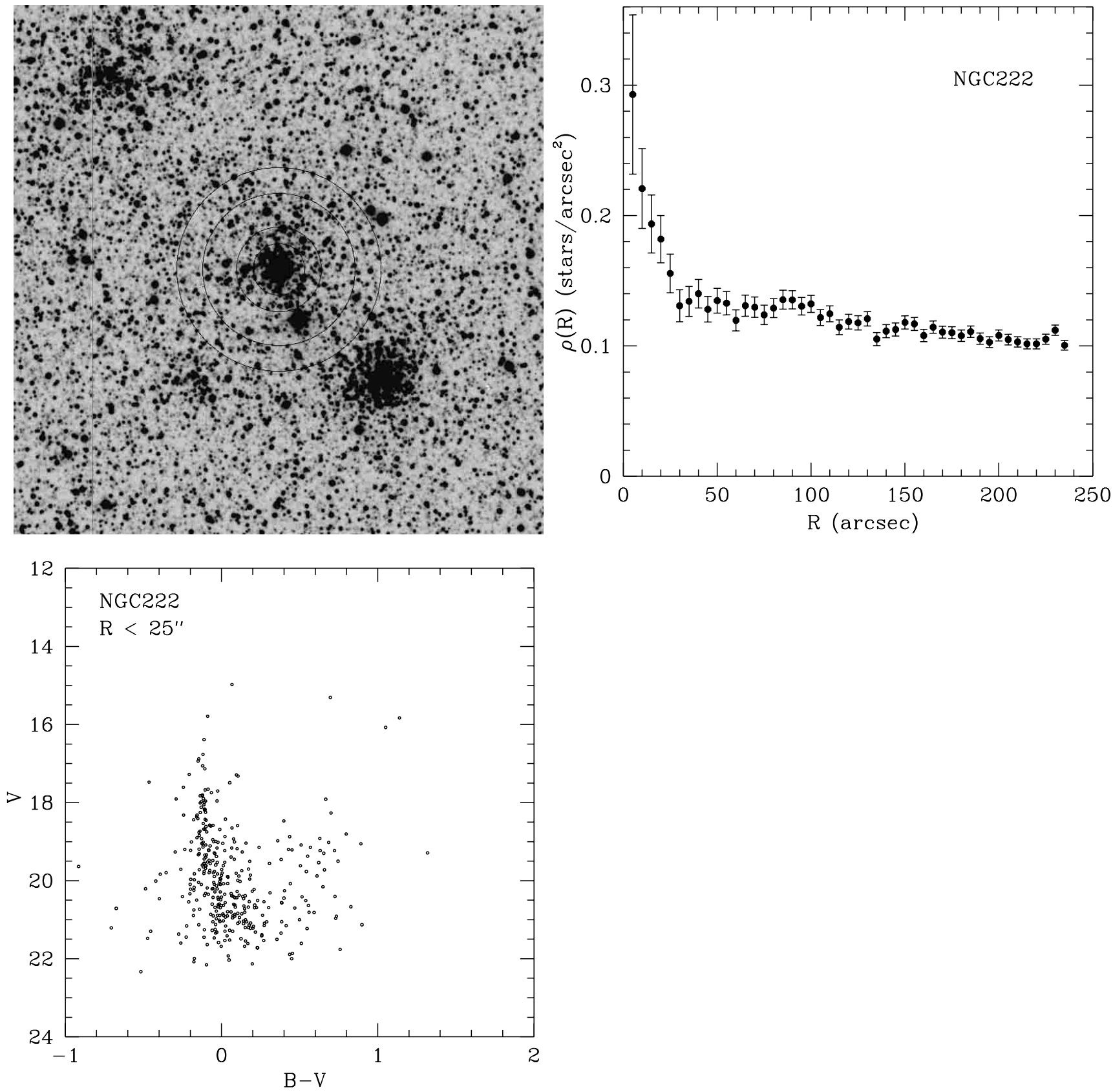

Fig. 20. In the left upper panel, the annuli division of NGC 222 region, of radius $15^{\prime \prime}, 25^{\prime \prime}, 45^{\prime \prime}$ and $60^{\prime \prime}$ respectively; in the right upper panel, the radial stellar distribution around the NGC 222 center. In the lower panel, the cluster CM diagram.

of the CMD of stars brighter than $V=21$ mag: Table 6 gives $N_{B}$ and $N_{R}$ for the various fields. A numerically consistent young population is present in L45, NGC 220 and NGC 458 fields: from respective CMDs (Figs. 16, 19 and 22) it appears that MS termination becomes fainter with increasing distance from the SMC center. L9, L13 and NGC 152 fields are located in SW region at $1.5^{\circ}-2^{\circ}$ from SMC center: they have detectably smaller $N_{B}$, but their CMDs (Figs. 14, 15 and 18) still show a young MS population, even if not with identical features. The L9 field CMD is morphologically similar to NGC 458 (MS termination $\sim 17.0 \mathrm{mag}$ ), while the NGC 152 field CMD presents a less developed MS, with a few stars brighter than $V=18.5$ mag. We have not performed this analysis on the NGC 643 field, that is located $4^{\circ}$ from SMC center in the outer SE region, since only 379 stars (see Fig. 23) brighter than $V=21$ mag have been identified. However, the very young population appears completely absent, in agreement with Gardiner \& Hatzidimitriou (1992) who found few bright MS in this region.

A comparison between the CMDs of studied clusters and the surrounding fields suggests that near the SMC Bar they are quite similar, with the youngest field component resembling cluster populations (see Figs. 16, 19 and 22), while in the SW region clusters appear older than the 

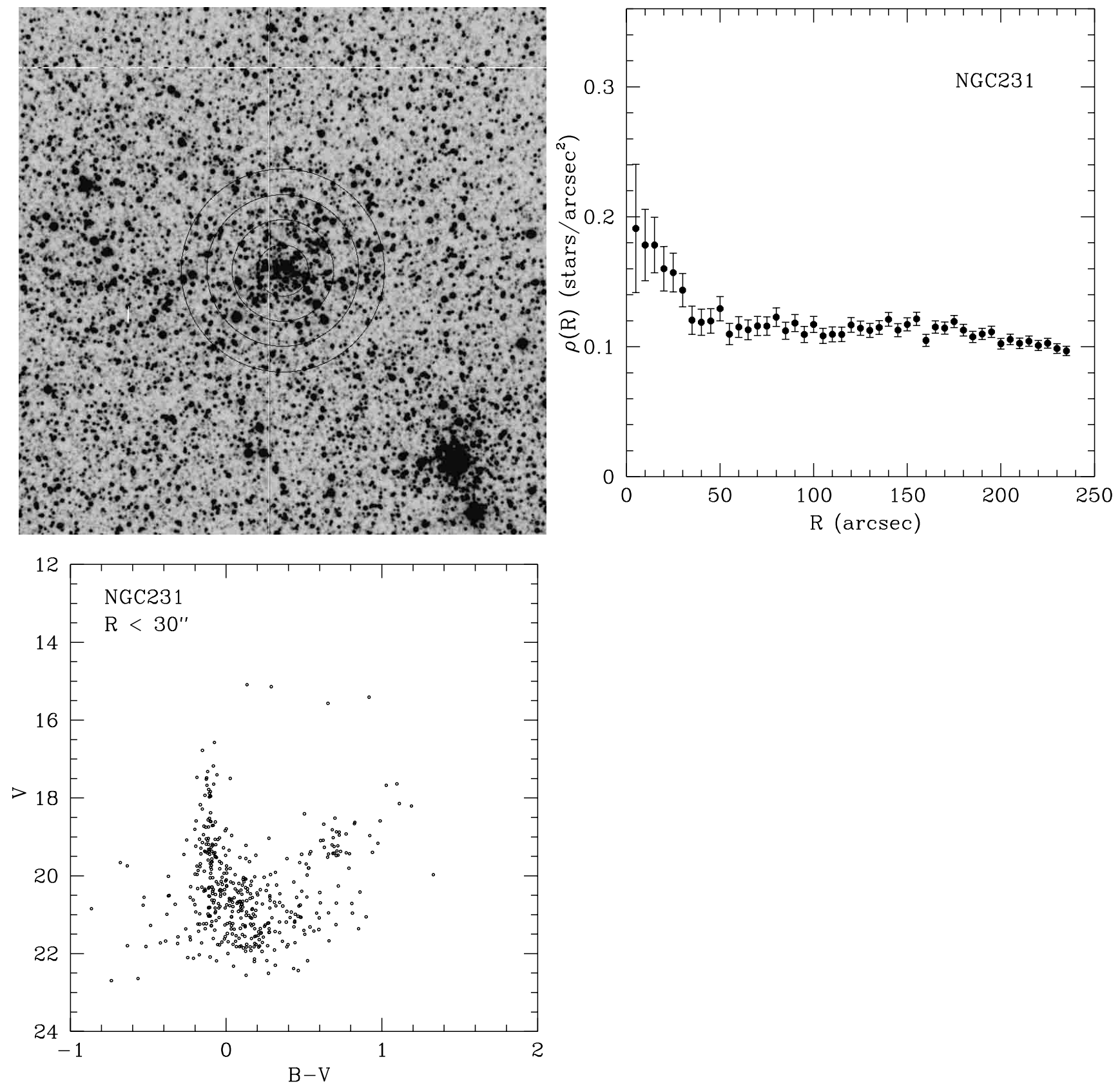

Fig. 21. In the left upper panel, the annuli division of NGC 231 region, of radius $15^{\prime \prime}, 30^{\prime \prime}, 45^{\prime \prime}$ and $60^{\prime \prime}$ respectively; in the right upper panel, the radial stellar distribution around the NGC 231 center. In the lower panel, the cluster CM diagram.

youngest field population. This is clearly evident in the L9 and NGC 152 cases (see Figs. 14 and 18).

\section{Field red clumps}

An evident feature of fields CMD is, except in the case of NGC 643, the occurrence of a well-defined red clump. This locus of the CM diagram, containing core helium-burning stars of intermediate and small mass, has captured the attention of many researchers as an alternative way to constrain the distance of extragalactic objects. Paczynski \& Stanek (1998) suggested the use of red clump mean absolute $I$ magnitude $\left(M_{I}\right)$ as a standard candle, since there was theoretical evidence that absolute luminosity of such core helium-burning stars depends only weakly on their age and metallicity (e.g. Seidel et al. 1987; Castellani et al. 1992).

The calibration of this method is based on the absolute I magnitude of clump stars in the solar neighbourhood with trigonometric parallaxes measured by the Hipparcos satellite. Using this method, Udalski et al. (1998) and Stanek et al. (1998) found distances for the LMC and SMC 15\% lower than values derived from other standard candles (such as Cepheids or RR Lyrae). However, recent theoretical investigations (Girardi et al. 1998; Girardi \& Salaris 2001) suggest that population effects can influence $M_{I}$ up to $0.4 \mathrm{mag}$, so results based on the red clump 

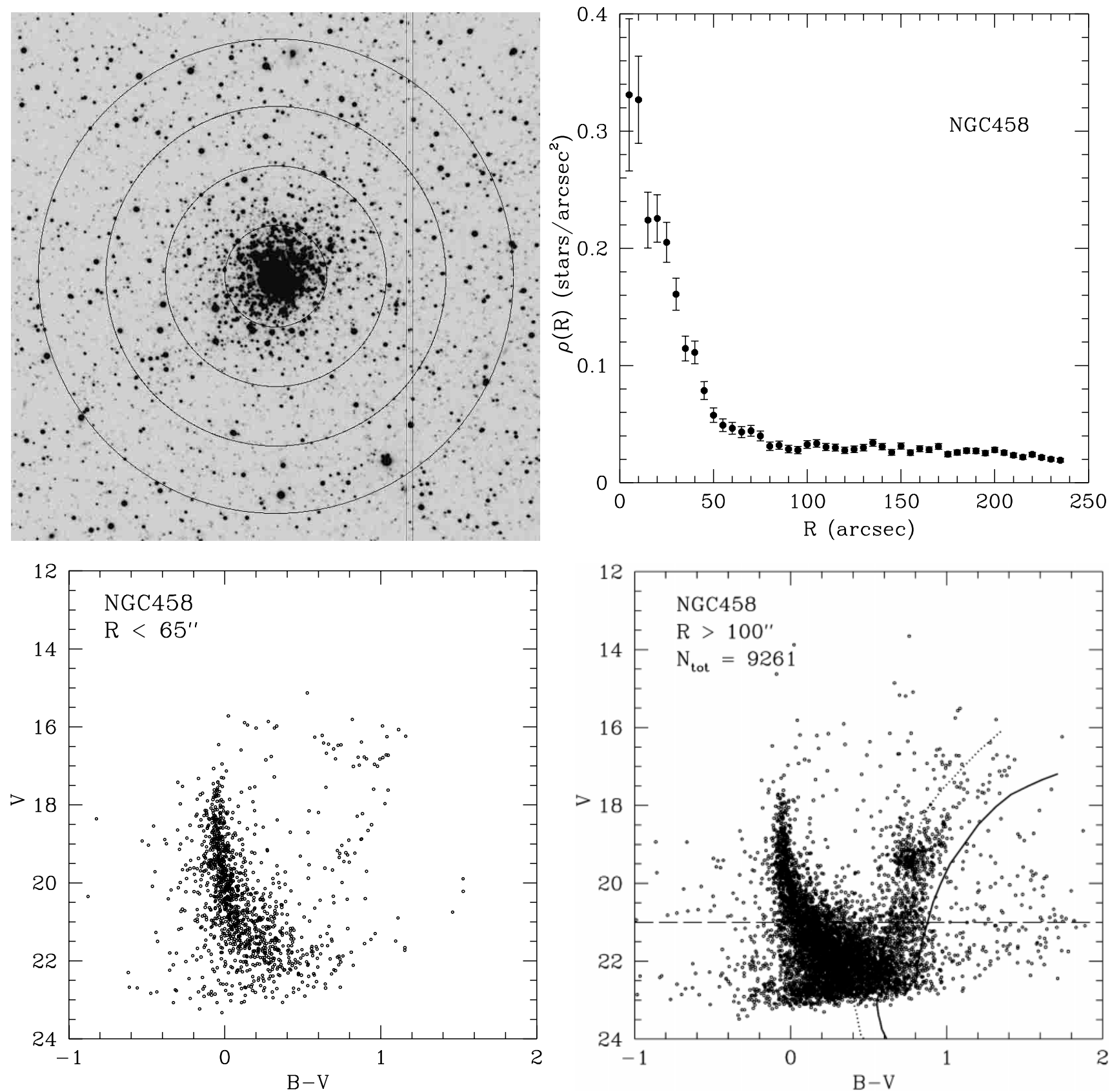

Fig. 22. In the left upper panel, the annuli division of NGC 458 region, of radius $30^{\prime \prime}, 65^{\prime \prime}, 100^{\prime \prime}$ and $140^{\prime \prime}$ respectively; in the right upper panel, the radial stellar distribution around the NGC 458 center. In the lower panels, the cluster and the field CM diagrams (we have reported 4550 stars fainter than $V=21 \mathrm{mag}$ ); ridge lines of M 15 (dotted line) and 47 Tuc (solid line) clusters are overimposed (see Sect. 4 for details).

method should be regarded with suspicion if these effects have been neglected. Unfortunately, population effects are not easy to estimate, because they depend on the assumptions of the history star formation rate $(S F R)$ and on the age-metallicity relation $(A M R)$.

To add data to such a relevant problem we made use of our sample to derive mean $V$ magnitude and colour $\left(<V_{\mathrm{RC}}>\right.$ and $\left.<(B-V)_{\mathrm{RC}}>\right)$ for red clumps in the investigated field regions. For each Cloud, we have defined a colour-magnitude box containing red clump: $0.60 \leq(B-V) \leq 1.15$ and $18.5 \leq V \leq 20.0$ for LMC,
$0.45 \leq(B-V) \leq 1.00$ and $18.7 \leq V \leq 20.2$ for $\mathrm{SMC}$ (examples are given in Fig. 26). For each field we have then constructed two histograms, the first representing the number of stars contained in bins of 0.05 mag with varying magnitude, the second representing the number of stars contained in colour bins of $0.02 \mathrm{mag}$ with varying colour. $<V_{\mathrm{RC}}>$ has been determined by fitting the histograms with a function of the form (Girardi \& Salaris 2001):

$N(V)=a+b V+c V^{2}+d \exp \left[-\frac{\left(V-<V_{\mathrm{RC}}>\right)^{2}}{2 \sigma_{V}^{2}}\right]$ 

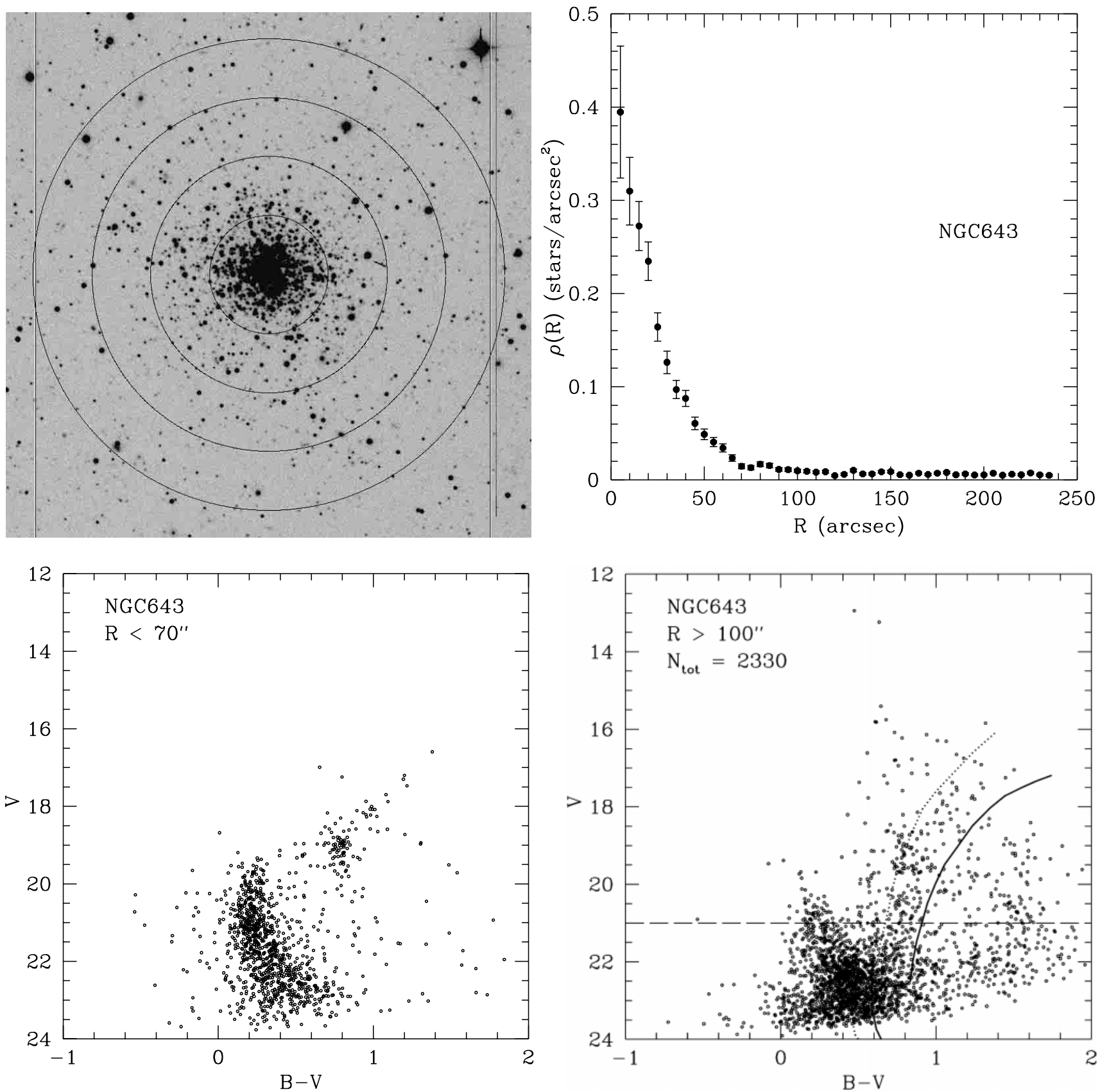

Fig. 23. In the left upper panel, the annuli division of NGC 643 region, of radius $35^{\prime \prime}, 70^{\prime \prime}, 105^{\prime \prime}$ and $140^{\prime \prime}$ respectively; in the right upper panel, the radial stellar distribution around the NGC 643 center. In the lower panels, the cluster and the field CM diagrams (we have reported all field star, and only 379 are brighter than $V=21 \mathrm{mag}$ ); ridge lines of $\mathrm{M} 15$ (dotted line) and 47 Tuc (solid line) clusters are overimposed (see Sect. 4 for details).

where $\sigma_{V}$ gives an estimate of the sharpness of the magnitude distribution of clump stars. In a similar way we have determined $<(B-V)_{\mathrm{RC}}>$. An example of the fits is given in Fig. 27.

Table 7 gives the derived values, whereas Fig. 28 shows the location of the clumps in the $V,(B-V)$ plane. In the LMC case, red clumps seems to concentrate in magnitude at $V \sim 19.25 \mathrm{mag}$, except for the NGC 2155 clump, which appears noticeably brighter $(V=19.00 \mathrm{mag})$ and slightly bluer. SMC clumps present an analogous concentration around $V \sim 19.48$ mag. A most evident difference in mean locations is that SMC red clumps are bluer $(\sim 0.10$ $0.15 \mathrm{mag}$ ) than for the LMC, in good agreement with theoretical predictions about metallicity effects (see Girardi 1999).

From the tabulation by Girardi \& Salaris (2001) one finds that, if the field of the two Clouds have similar histories (but different metallicities), SMC clumps at $Z=0.004$ are expected to be more luminous than clumps in the LMC $(Z=0.008)$ by $0.11-0.14 \mathrm{mag}$. By relying on such a figure one would derive a difference in distance moduli between the two Clouds of the order of 0.35 mag. Along a similar 
Table 4. Selected quantities for the LMC and SMC clusters arranged in order of increasing age.

\begin{tabular}{|c|c|c|c|c|c|c|}
\hline & Cluster & SWB class & $R(\operatorname{arcsec})$ & $N_{\text {star }}$ & $V_{\mathrm{TO}}(\mathrm{mag})$ & age (Myr) \\
\hline \multirow[t]{6}{*}{ LMC } & KMHK265 & $\ldots$ & 30 & 303 & 16.5 & $50 \div 100$ \\
\hline & NGC 1902 & II & 40 & 440 & 17 & $100 \div 150$ \\
\hline & KMHK264 & $\ldots$ & 30 & 241 & 17.5 & $150 \div 200$ \\
\hline & NGC 1777 & IV B & $25 \div 70$ & 804 & 19.5 & $700 \div 800$ \\
\hline & IC 2146 & $\mathrm{~V}$ & 60 & 2023 & 20.25 & $1200 \div 1500$ \\
\hline & NGC 2155 & VI & $16 \div 50$ & 1085 & 20.5 & $1500 \div 2000$ \\
\hline \multirow[t]{10}{*}{$\mathrm{SMC}$} & NGC 299 & $\ldots$ & 25 & 271 & 14.5 & $15 \div 20$ \\
\hline & NGC 220 & III & 30 & 511 & 16.5 & $70 \div 100$ \\
\hline & NGC 222 & II-III & 25 & 361 & 16.5 & $70 \div 100$ \\
\hline & NGC 231 & $\ldots$ & 30 & 449 & 16.5 & $70 \div 100$ \\
\hline & NGC 458 & III & 65 & 1288 & 17.0 & $100 \div 150$ \\
\hline & L45 & $\ldots$ & 30 & 334 & 17.0 & $100 \div 150$ \\
\hline & L13 & . & 35 & 300 & 19.25 & $450 \div 550$ \\
\hline & NGC 643 & $\ldots$ & 70 & 1127 & 19.5 & $600 \div 700$ \\
\hline & L9 & .. & 35 & 374 & $20.25 \div 20.5$ & $1000 \div 1300$ \\
\hline & NGC 152 & IV B & 60 & 1862 & $20.25 \div 20.5$ & $1000 \div 1300$ \\
\hline
\end{tabular}

Table 5. Results of the star counts for the selected regions, containing 1950 stars, of observed LMC fields, named as the included cluster. We assume, as statistical fluctuation on these numbers, the Poisson error.

\begin{tabular}{cccc}
\hline \hline Field & $N_{B}$ & $N_{R}$ & $N_{B} / N_{R}$ \\
\hline KMHK264 & $1212 \pm 35$ & $738 \pm 27$ & $1.64 \pm 0.11$ \\
NGC 1902 & $1296 \pm 36$ & $654 \pm 26$ & $1.98 \pm 0.13$ \\
NGC 2155 & $1202 \pm 35$ & $748 \pm 27$ & $1.61 \pm 0.11$ \\
NGC 1777 & $910 \pm 30$ & $1040 \pm 32$ & $0.88 \pm 0.06$ \\
IC 2146 & $930 \pm 30$ & $1020 \pm 32$ & $0.91 \pm 0.06$ \\
\hline
\end{tabular}

route, one finds that neither reddening nor metallicity can account for the peculiar behaviour of the NGC 2155 field, and apparently we remain with the only solution that these stars are really closer to us.

\subsection{Comparison between field and cluster red clumps}

Finally, we have adopted the already quoted procedure to also derive mean magnitudes and colours of the cluster red clumps, as given in Table 8 . Contrary to the field, in
Table 6. Results of the star counts for the selected regions, containing 1950 stars, of observed SMC fields, named as the included cluster. We assume, as statistical fluctuation on these numbers, the Poisson error.

\begin{tabular}{cccc}
\hline \hline Field & $N_{B}$ & $N_{R}$ & $N_{B} / N_{R}$ \\
\hline L45 & $1124 \pm 34$ & $826 \pm 29$ & $1.36 \pm 0.09$ \\
NGC 220 & $1102 \pm 33$ & $848 \pm 29$ & $1.30 \pm 0.08$ \\
NGC 458 & $1224 \pm 35$ & $726 \pm 27$ & $1.69 \pm 0.11$ \\
L9 & $876 \pm 30$ & $1074 \pm 33$ & $0.82 \pm 0.05$ \\
L13 & $756 \pm 27$ & $1194 \pm 35$ & $0.63 \pm 0.04$ \\
NGC 152 & $610 \pm 25$ & $1340 \pm 37$ & $0.46 \pm 0.03$ \\
\hline
\end{tabular}

this case one is dealing with a "snapshot" depicting the luminosity of He burning stars for a given (cluster) age. However, the comparison with the correspondent values for the field as given in Table 7 show few differences, in many cases possibly connected with the large dispersion and poor statistics of the observational data. The only relevant evidence is for NGC 643, whose clump appears about 0.5 mag brighter than the mean magnitude of the other clumps in the SMC field. This could be the effect of a younger age, but it could be also attributed to a distance 


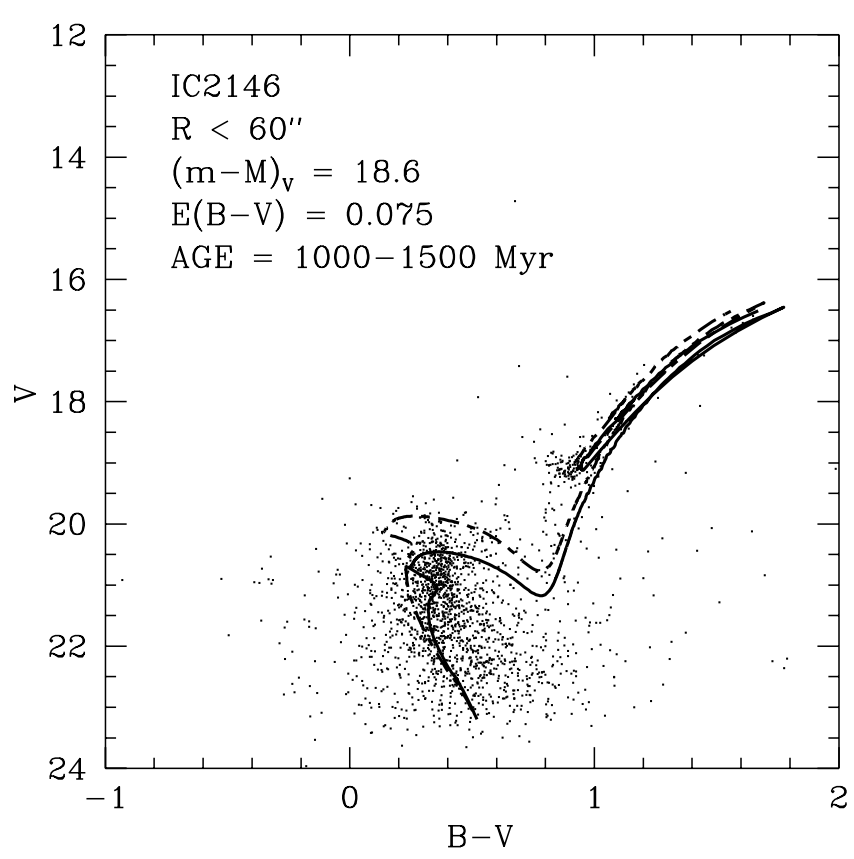

Fig. 24. Isochrone fitting for IC 2146: solid and dashed line show the 1500 and 1000 Myr isochrones respectively.

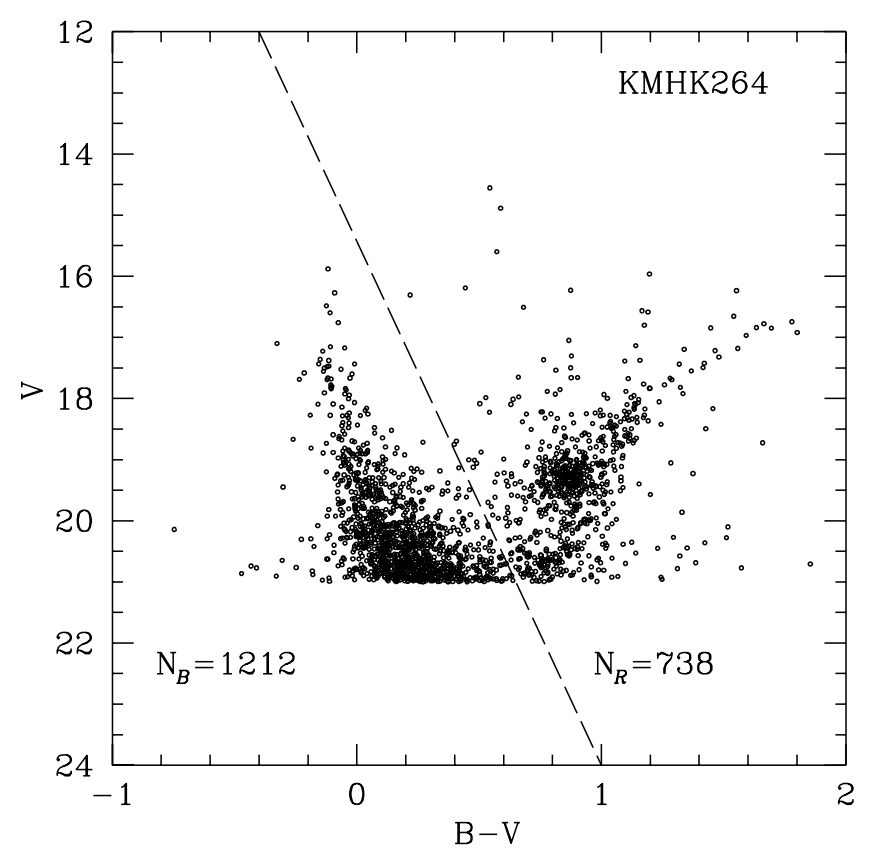

Fig. 25. Division in MS and evolved red stars regions of KMHK264 field CMD containing 1950 stars brighter than $V=$ 21 mag.

Table 7. Mean values and statistical errors for field red clump. $N_{\text {star }}^{\mathrm{RC}}$ gives the total number of stars in the box.

\begin{tabular}{cccccc}
\hline \hline Field & $N_{\mathrm{star}}^{\mathrm{RC}}$ & $<V_{\mathrm{RC}}>$ & $\sigma_{\mathrm{RC}}^{(V)}$ & $\left\langle(B-V)_{\mathrm{RC}}>\right.$ & $\sigma_{\mathrm{RC}}^{(B-V)}$ \\
\hline LMC & & & & & \\
KMHK264 & 1789 & 19.29 & 0.15 & 0.875 & 0.050 \\
NGC 1902 & 1284 & 19.28 & 0.13 & 0.880 & 0.045 \\
NGC 2155 & 542 & 19.00 & 0.14 & 0.860 & 0.041 \\
NGC 1777 & 654 & 19.28 & 0.15 & 0.915 & 0.033 \\
IC 2146 & 514 & 19.22 & 0.07 & 0.895 & 0.037 \\
\hline SMC & & & & & \\
L45 & 2914 & 19.47 & 0.17 & 0.780 & 0.061 \\
NGC 220 & 4362 & 19.48 & 0.14 & 0.725 & 0.057 \\
NGC 458 & 445 & 19.42 & 0.09 & 0.770 & 0.039 \\
L9 & 710 & 19.52 & 0.17 & 0.735 & 0.037 \\
L13 & 1373 & 19.48 & 0.10 & 0.745 & 0.055 \\
NGC 152 & 1545 & 19.45 & 0.20 & 0.750 & 0.037 \\
\hline & & & & & \\
\hline
\end{tabular}

effect, NGC 643 (located $4^{\circ}$ far from SMC center) being closer to us than the SMC.

\section{Conclusions}

In this paper we have presented BV CCD photometry for 6 star clusters in the LMC, 10 clusters in the SMC and for surrounding stellar fields obtained from observations with the 1.54-m Danish Telescope. After having described the principal features of cluster CMDs, we have discussed field CMDs separately for the two Clouds. A number of significant results have emerged from this study. We found evidence that star formation events in the recent past have not been the same in different LMC outer regions: near the 

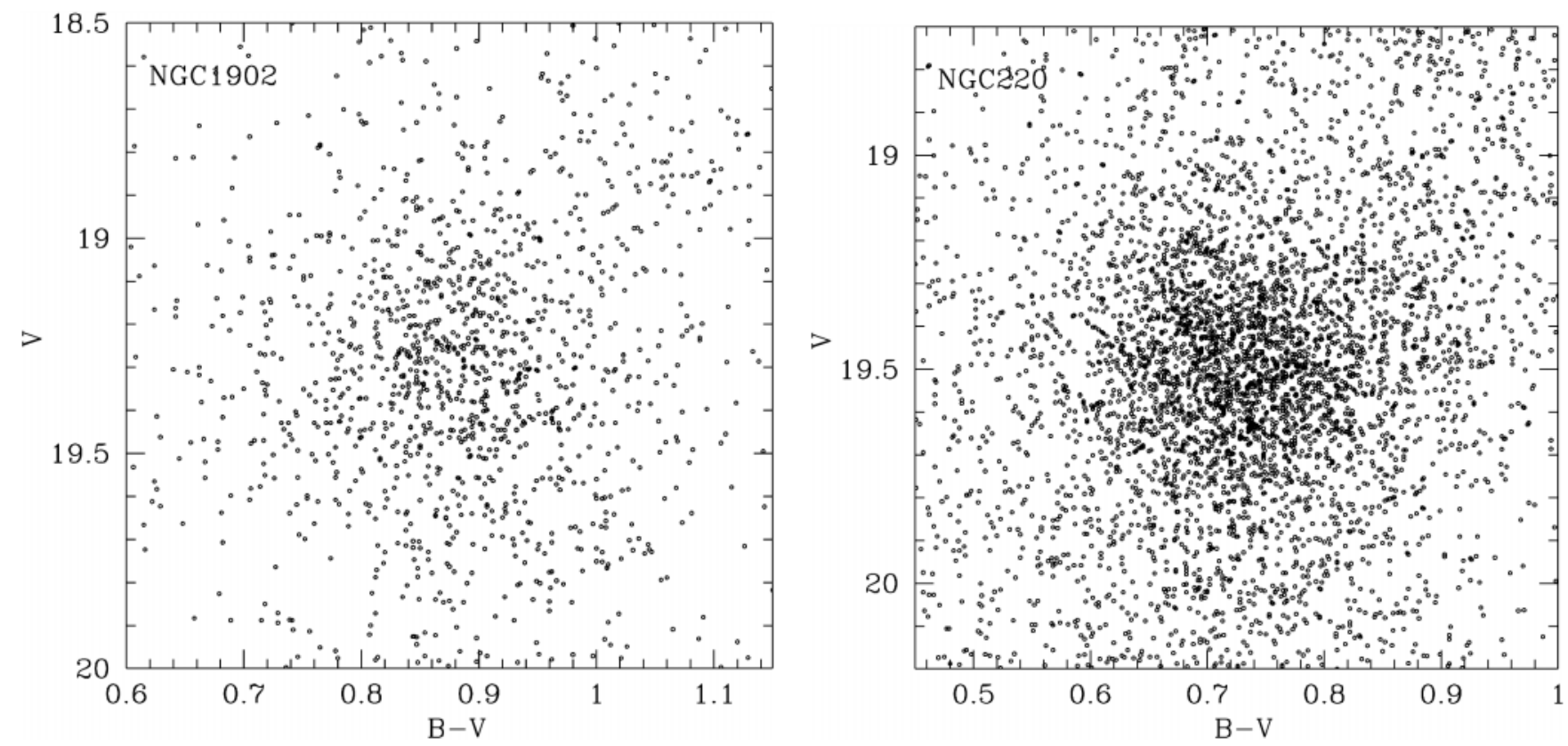

Fig. 26. CMD boxes containing red clump stars for NGC 1902 LMC field (left panel) and for NGC 220 SMC field (right panel). Similar boxes was defined for the other fields of our sample.
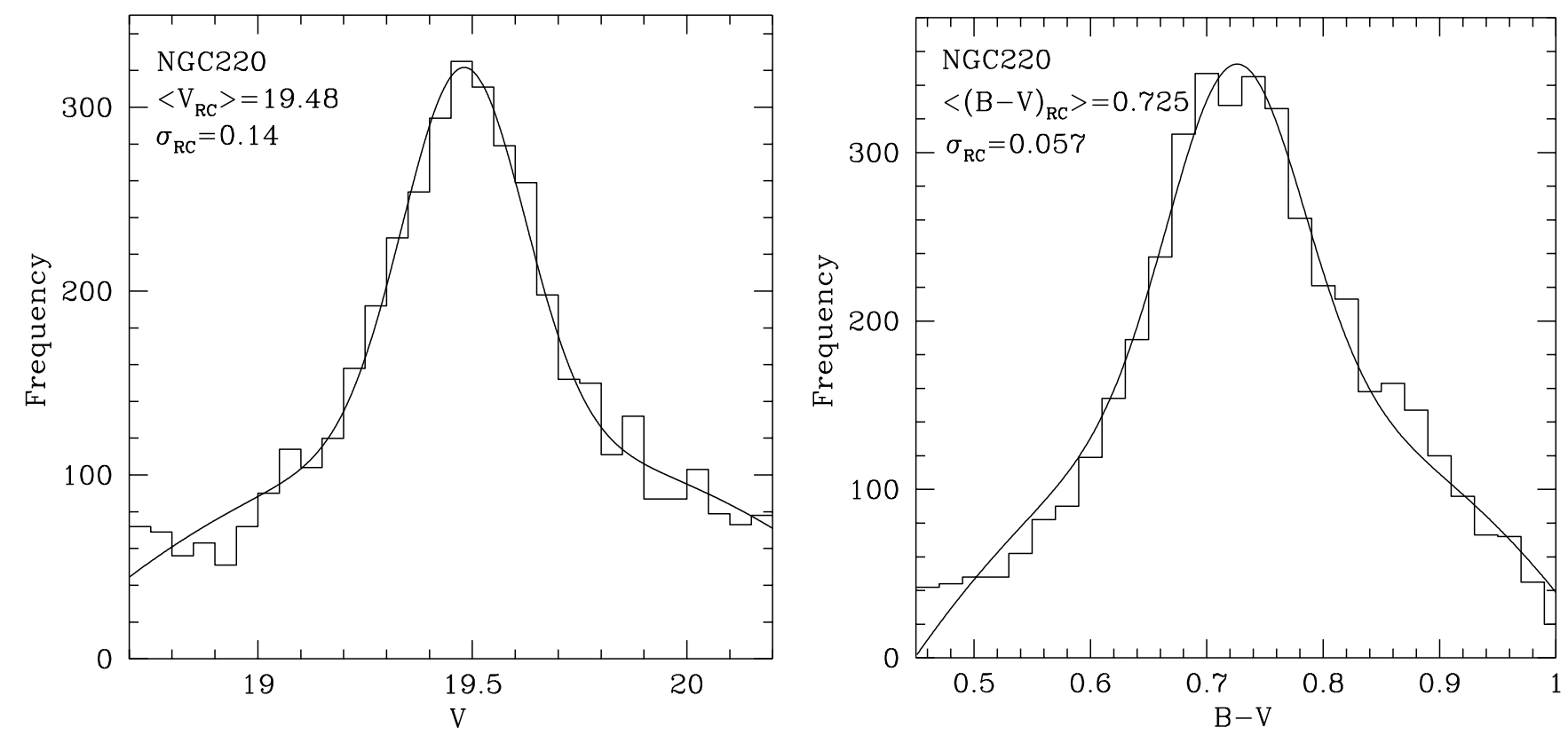

Fig. 27. Results of the fitting procedure on NGC 220 field clump histograms in magnitude (left panel) and colour (right panel). Similar analysis has been performed on all the other red clumps.

Bar a young population (up to $50-100 \mathrm{Myr}$ ) is present, whereas southern fields do not show this component.

For the SMC, we found that a young population (up to $50-100 \mathrm{Myr}$ ) is present in each of the analysed fields (except NGC 643's field), even if it decreases in numerical abundance with increasing distance from the SMC center. Towards $1.5^{\circ}-2^{\circ}$ from SMC center, the age of the youngest component tends to increase. Near the SMC Bar, clusters resemble quite well the youngest field component morphology, where in the SW region they appears older than the surrounding field.
We have also determined mean positions in the $V$, $(B-V)$ plane of red clumps of our fields, finding that the NGC 2155 field clump is $\sim 0.25$ mag brighter than the others in the LMC located at $V \sim 19.25$ mag, whereas all SMC clumps tends to concentrate in magnitude at $V \sim 19.48$ mag. SMC clumps are 0.10-0.15 mag bluer than LMC, in agreement with theoretical predictions on the effects of stellar metallicity.

Acknowledgements. We are very grateful to Dr. S. Degl'Innocenti, Dr. P. G. Prada Moroni and Dr. M. Marconi for providing us the set of isochrones used in this work. 

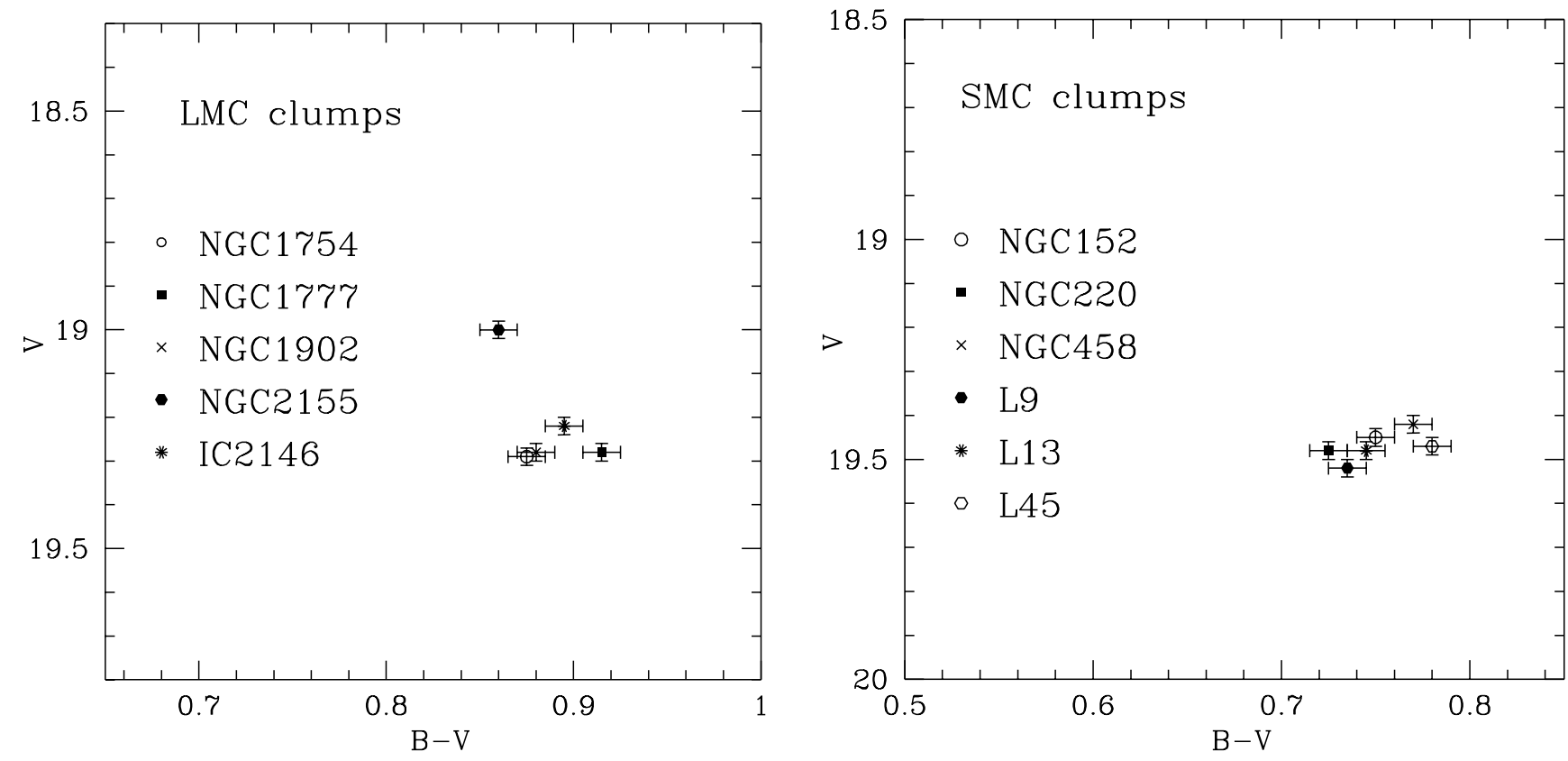

Fig. 28. Mean positions in the $V,(B-V)$ plane of LMC (left panel) and SMC (right panel) studied red clumps.

Table 8. Mean magnitudes $<V_{\mathrm{RC}, \mathrm{c}}>$ and colours $<(B-V)_{\mathrm{RC}, \mathrm{c}}>$ of the cluster red clumps, with $\sigma_{\mathrm{RC}, \mathrm{c}}^{(V)}$ and $\sigma_{\mathrm{RC}, \mathrm{c}}^{(B-V)}$ giving estimates of the clump sharpness in magnitude and colour, respectively.

\begin{tabular}{ccccc}
\hline \hline Cluster & $<V_{\mathrm{RC}, \mathrm{c}}>$ & $\sigma_{\mathrm{RC}, \mathrm{c}}^{(V)}$ & $\left\langle(B-V)_{\mathrm{RC}, \mathrm{c}}>\right.$ & $\sigma_{\mathrm{RC}, \mathrm{c}}^{(B-V)}$ \\
\hline LMC & & & & \\
NGC 2155 & 18.87 & 0.12 & 0.85 & 0.07 \\
NGC 1777 & 19.32 & 0.21 & 0.83 & 0.10 \\
IC 2146 & 19.15 & 0.16 & 0.90 & 0.06 \\
\hline SMC & & & & 0.08 \\
L9 & 19.57 & 0.19 & 0.75 & 0.08 \\
NGC 152 & 19.52 & 0.16 & 0.75 & 0.06 \\
NGC 643 & 19.00 & 0.20 & 0.80 & \\
\hline
\end{tabular}

We also thank the referee, Dr. M. G. Lee, whose remarks allowed us to significantly improve the manuscript.

This research has made use of the SIMBAD database operated by CDS, Strasbourg, France. This work is supported by Italian Ministry of University, Scientific Research and Technology (MURST) within the Cofin2000 project "Stellar Observables of Cosmological Relevance".

\section{References}

Arp, H. 1959, AJ, 64, 175

Bertelli, G., Mateo, M., Chiosi, C., \& Bressan, A. 1992, AJ, 388,400

Brocato, E., Di Carlo, E., \& Menna, G. 2001, A\&A, 374, 523

Castellani, V., Chieffi, A., \& Straniero, O. 1992, ApJS, 78, 517

Dolphin, A. E., Walker, A. R., Hodge, P. W., et al. 2001, ApJ, accepted [astro-ph/0107164]
Durrell, P. R., \& Harris, W. E. 1993, AJ, 105, 1420

Gardiner, L. T., \& Hatzidimitriou, D. 1992, MNRAS, 257, 195

Geha, M., Holtzman, J. A., Mould, J. R., et al. 1998, AJ, 115, 1045

Girardi, L. 1999, MNRAS, 308, 818

Girardi, L., Groenewegen, M. A. T., Weiss, A., \& Salaris, M. 1998, MNRAS, 301, 149

Girardi, L., \& Salaris, M. 2001, MNRAS, 323, 109

Graham, J. A. 1982, PASP, 94, 244

Harris, W. E. 1996, AJ, 112, 1487 http://physun.physics.mcmaster.ca/Globular.html

Hodge, P. W. 1981, ApJ, 247, 894

Landolt, A. U. 1992, AJ, 104, 340

Mateo, M., \& Hodge, P. 1985, PASP, 97, 753

Matteucci, A. 2001, Diploma Thesis, Università degli Studi di Pisa

Olszewski, E. W., Suntzeff, N. B., \& Mateo, M. 1996, ARA\&A, 34,511 
Paczynski, B., \& Stanek, K. Z. 1998, ApJ, 494, L219

Rich, R. M., Shara, M., Fall, S. M., \& Zurek, D. 2000, AJ, 119, 197

Santos, J. F. C., Jr., Piatti, A. E., Clariá, J. J., et al. 1999, AJ, 117,2841

Sarajedini, A. 1998, AJ, 116, 738

Schlegel, D. J., Finkbeiner, D. P., \& Davis, M. 1998, ApJ, 500, 525

Searle, L., Wilkinson, A., \& Bagnuolo, W. G. 1980, ApJ, 239, 803

Seidel, E., Demarque, P., \& Weinberg, D. 1987, ApJS, 63, 917

Sosin, C., Piotto, G., Djorgovski, et al. 1997, in Advances in Stellar Evolution, ed. R. T. Rood, \& A. Renzini (Cambridge: Cambridge University Press), 92

Stanek, K. Z., Zaritsky, D., \& Harris, J. 1998, ApJ, 500, L141 Stetson, P. B. 1994, PASP, 106, 250
Udalski, A., Szymanski, M., Kubiak, M., et al. 1998, Acta Astron., 48, 1

Vallenari, A., Chiosi, C., Bertelli, G., \& Ortolani, S. 1996a, A\&A, 309, 358

Vallenari, A., Chiosi, C., Bertelli, G., Aparicio, A., \& Ortolani, S. 1996b, A\&A, 309, 367

Van den Bergh, S. 1981, A\&AS, 46, 79

Van den Bergh, S. 1991, in The Magellanic Clouds, ed. R. Haynes, \& D. Milne (Kluwer, Dordrecht, Holland), IAU Symp. No. 148, 161

Westerlund, B. E. 1997, in The Magellanic Clouds, ed. Cambridge press, 229

Westerlund, B. E., Linde, P., \& Lyngå, G. 1995, A\&A, 298, 39

Westerlund, B. E., Lundgren, K., Petterson, B., \& Koziej, E. 1998, A\&A, 339, 385 